\title{
THE EFFECT OF VARIOUS LEVELS OF DIETARY STARCH ON GLYCOGEN REPLENISHMENT IN THE LIGHT WORKING HORSE
}

\author{
A Thesis \\ by \\ COURTNEY ANN PHILLIPS
Submitted to the Office of Graduate and Professional Studies of Texas A\&M University
in partial fulfillment of the requirements for the degree of \\ MASTER OF SCIENCE
}

Chair of Committee, Clay Cavinder

Co-Chair of Committee, Dennis Sigler

Committee Member, James Fluckey

Head of Department, $\quad$ H. Russell Cross

December 2015

Major Subject: Animal Science

Copyright 2015 Courtney Ann Phillips 


\section{ABSTRACT}

Nine Quarter Horses (2 to $7 \mathrm{yr} ; 409$ to $494 \mathrm{~kg} \mathrm{BW}$ ) were used in a 3 x 3 Latin square with replication study lasting $105 \mathrm{~d}$ to determine the effect of various levels of dietary starch on glycogen replenishment in the light working horse. Horses were fed 1 $\% \mathrm{BW} / \mathrm{d}$ in Coastal Bermudagrass hay with remaining calories met by a high starch (HS), medium starch (MS), or low starch (LS) concentrate. After a $7 \mathrm{~d}$ washout period, horses were transitioned to 1 of the 3 diets over $7 \mathrm{~d}$ for a $14 \mathrm{~d}$ treatment period where they were then worked to fatigue in a standardized exercise test (SET). Total diets provided an average of 1,206.67, 844.61, and $263.13 \mathrm{~g}$ of starch/d in HS, MS, and LS, respectively. Horses were lightly exercised for $30 \mathrm{~min} 3 \mathrm{~d} / \mathrm{wk}$. The SET consisted of a 30 min trot in a panel exerciser, followed by 27 min of an incremental high-intensity work on a treadmill.

Skeletal muscle biopsies were taken from the biceps femoris at rest, immediately after the SET, and 24 and $48 \mathrm{~h}$ post exercise. Samples were submerged in liquid nitrogen and stored at $-80^{\circ} \mathrm{C}$ until glycogen analysis using a commercial kit. Venous blood samples were taken at rest, immediately post exercise, 10 min after recovery, and $24 \mathrm{~h}$ post exercise. Data was analyzed using Proc Mixed (SAS) program.

High starch had higher resting muscle glycogen concentration $(P=0.009)$ than MS (10.25 vs. $8.28 \mu \mathrm{g} / \mathrm{mg}$ wet wt). Low starch had higher glycogen concentration $24 \mathrm{~h}$ post $(\mathrm{P}=0.04)$ than HS $(9.52$ vs. $7.68 \mu \mathrm{g} / \mathrm{mg}$ wet wt $)$. High starch utilized more glycogen than MS or LS. A slight reduction in glycogen post exercise for MS and LS 
indicated that fat or protein may have been used as substrate for exercise. Results indicated that feeding 1,206.67 $\mathrm{g}$ starch/d did not yield an advantage in recovery time over a MS or LS diet. Energy expenditure during the SET yielded similar $(\mathrm{P}=0.98)$ blood lactate concentrations, resulting in the formation of a prediction equation of $\mathrm{y}=$ $0.002 x^{2}-0.3102 x+6.6874$ 


\section{DEDICATION}

This thesis is dedicated to my parents, Mark and Diana Phillips. From a young age I was taught to be independent, hardworking, and to strive for my dreams. Their continued support and love made my educational endeavors possible. 


\section{ACKNOWLEDGEMENTS}

I would like to thank my co-chair of committee Dr. Sigler for his guidance and support throughout the course of this research. A special thank you goes to my chair of committee, Dr. Cavinder, my mentor throughout my time at Texas A\&M University. I would also like to thank committee member Dr. Fluckey and his graduate students for their assistance with lab analysis.

Thank you to all of my friends and fellow graduate students who helped me with lab analysis, guided me through my project, and helped when needed. I would like to extend my appreciation to my team of undergraduate students who assisted throughout the course of my project. I couldn't have asked for a harder working group of students who went above and beyond the call of duty to make this project not only successful, but fun. 


\section{NOMENCLATURE}

\section{Physical Units}

\begin{tabular}{|c|c|}
\hline $\mathrm{cm}$ & Centimeter \\
\hline $\mathrm{dL}$ & Deciliter \\
\hline $\mathrm{g}$ & Gram \\
\hline $\mathrm{kg}$ & Kilogram \\
\hline $\mathrm{kg}$ & Kilogram \\
\hline $\mathrm{km}$ & Kilometer \\
\hline $\mathrm{L}$ & Liter \\
\hline $\mathrm{m}$ & Meter \\
\hline Mcal & Megacalories \\
\hline$\mu g$ & Microgram \\
\hline$\mu \mathrm{L}$ & Microliters \\
\hline $\mathrm{mg}$ & Milligram \\
\hline $\mathrm{mL}$ & Milliliter \\
\hline $\mathrm{mm}$ & Milimeter \\
\hline mmol & Millimole \\
\hline $\mathrm{nm}$ & Nanometers \\
\hline wt & Weight \\
\hline \multicolumn{2}{|c|}{ Units of Time } \\
\hline$d$ & Day \\
\hline $\mathrm{h}$ & Hour \\
\hline
\end{tabular}




$\begin{array}{ll}\min & \text { Minute } \\ \mathrm{s} & \text { Second } \\ \mathrm{wk} & \text { Week } \\ \mathrm{yr} & \text { Year }\end{array}$

\section{Dietary}

\begin{tabular}{ll} 
ADF & Acid Detergent Fiber \\
AP & Alfalfa Pellets \\
BCS & Body Condition Score \\
BW & Body Weight \\
Ca & Calcium \\
CHO & Carbohydrate \\
CO & Corn Oil \\
DE & Dietary Energy \\
DM & Dry Matter \\
HPHF & High-Protein, High-Fat \\
HPLF & High-Protein, Low-Fat \\
FFA & Free Fatty Acids \\
FA & Fatty Acids \\
HS & High Starch \\
K & Potassium \\
LS & Low Starch \\
Mg & Magnesium \\
\hline
\end{tabular}


MS

Medium Starch

$\mathrm{N}$

$\mathrm{Na}$

NDF

NFC

NSC

$\mathrm{P}$

SFC

TDN

TG

VFA

Exercise

bpm

HR

$\mathrm{HR}_{\text {max }}$

RQ

SET

$\mathrm{VLA}_{4}$

$\mathrm{VO}_{2} \max$

Blood

A/G

$\mathrm{AB}$

Nitrogen

Sodium

Phosphorus

Triglycerides

Heart Rate

Albumin
Neutral Detergent Fiber

Non-Fiber Carbohydrate

Non-structural Carbohydrates

Steam Flaked Corn

Total Dietary Nutrients

Volatile Fatty Acid

Beats Per Minute

Maximum Heart Rate

Respiratory Quotient

Standardized Exercise Test

Velocity When Blood Lactate Reaches 4 mmol/L

Maximal Oxygen Uptake

Albumin/Globulin Ratio 
AST

$\mathrm{B}_{\mathrm{D}}$

$\mathrm{B}_{\mathrm{T}}$

BUN

CK

GGT

GLB

GLU

$\mathrm{SCr}$

SGOT

TSP

Statistical

CV

DLSM

LSM

Additional

ATP

EDTA

ELISA

G

GP

I.V.
Aspartate Aminotransferase

Bilirubin Direct

Total Bilirubin

Blood Urea Nitrogen

Creatine Kinase

Gamma-glutamyl Transpeptidase

Globulin

Glucose

Creatinine

Serum Glutamic-oxaloacetic Transaminase

Total Serum Protein

Covariance

Differences of Least Squares Means

Least Squares Means

Adenosine Triphosphate

Ethylenediaminetetraacetic Acid

Enzyme-Linked Immunosorbent Assay

Gauge (Needle)

Glycogen Phosphorylase

Intravenous 


$\begin{array}{ll}\text { LDH } & \text { Lactate Dehydrogenase } \\ \text { LI } & \text { Large Intestine } \\ \text { LPL } & \text { Lipoprotein Lipase } \\ \text { NG } & \text { Nasogastric Tube } \\ \text { PAS } & \text { Periodic Acid-Schiff Stain } \\ \text { PFK } & \text { Phosphofructokinase } \\ \text { rpm } & \text { Revolutions Per Minute } \\ \text { SDH } & \text { Succinic Dehydrogenase } \\ \text { SI } & \text { Small Intestine }\end{array}$




\section{TABLE OF CONTENTS}

Page

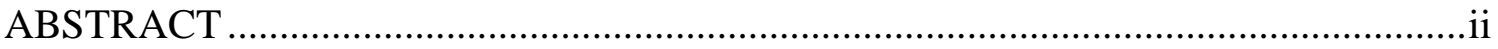

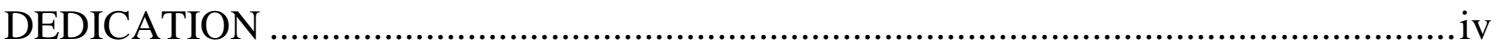

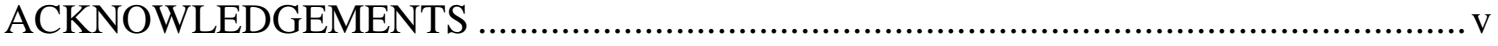

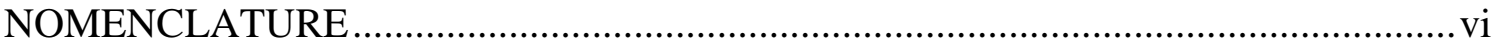

LIST OF FIGURES ...........................................................................................

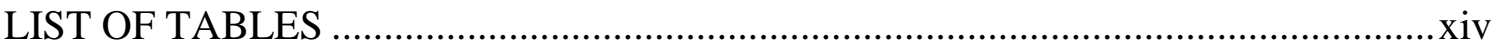

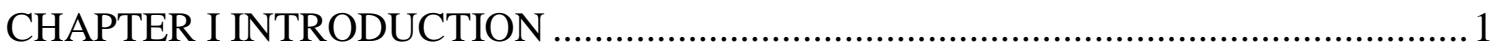

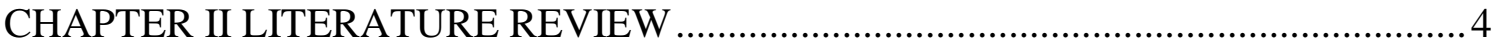

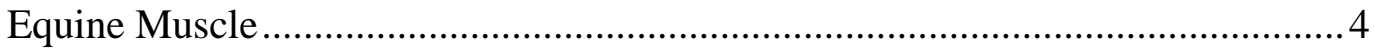

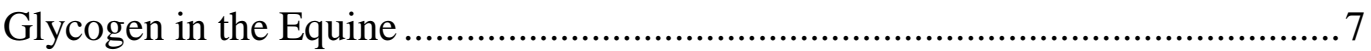

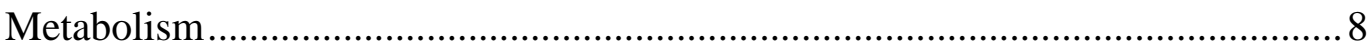

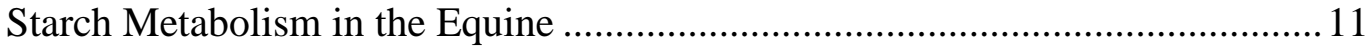

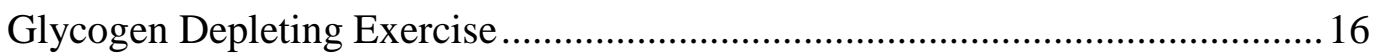

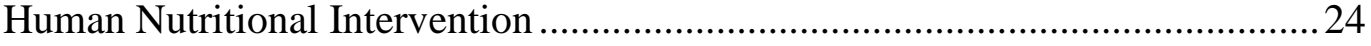

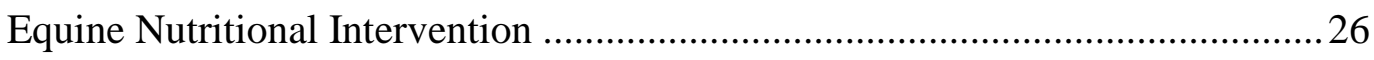

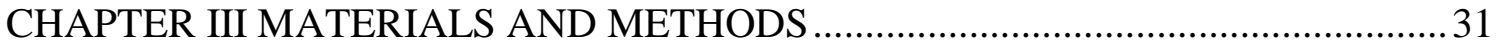

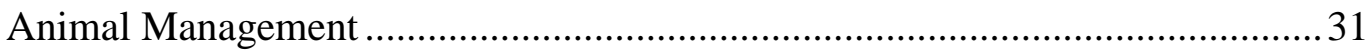

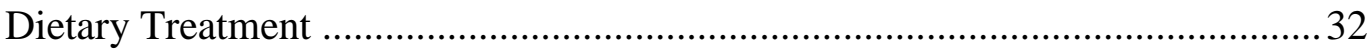

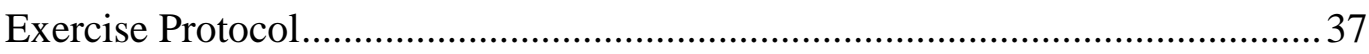

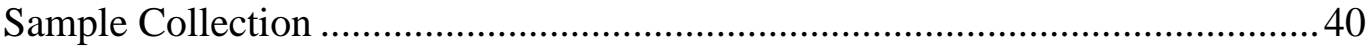

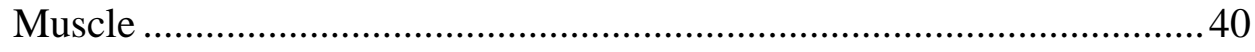

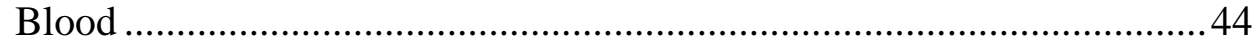

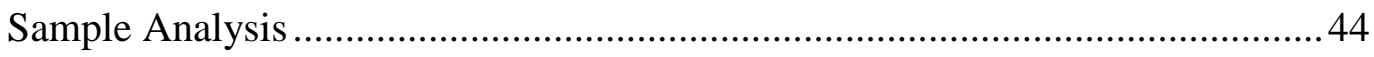

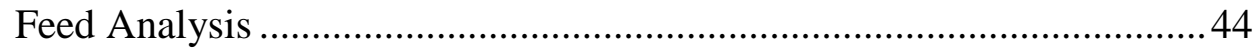

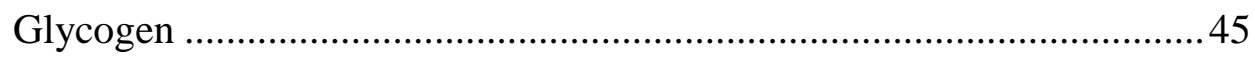

Blood ................................................................................ 47 
Statistical Analysis

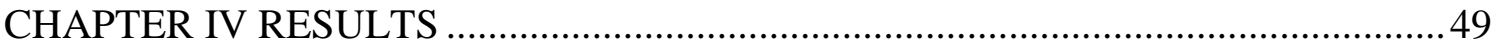

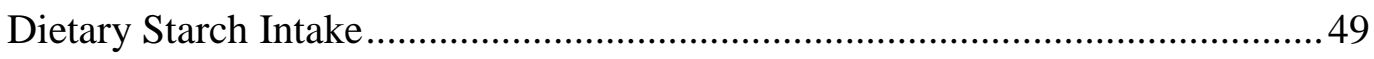

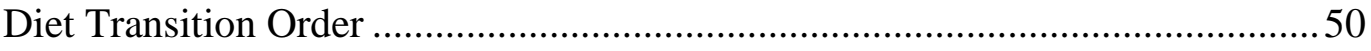

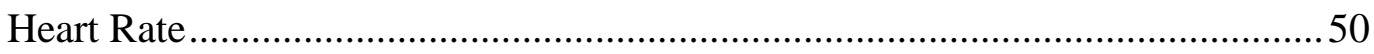

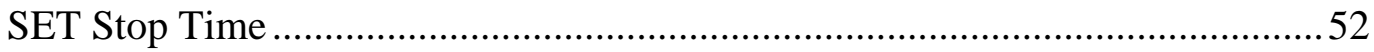

Skeletal Muscle Glycogen Concentration ...........................................................53

Glycogen Assay Kit Plate Internal Controls .......................................................57

Blood Markers .............................................................................................. 57

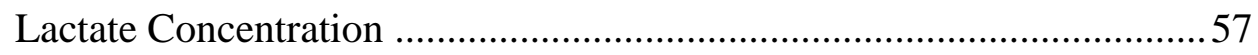

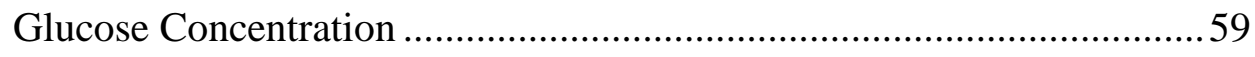

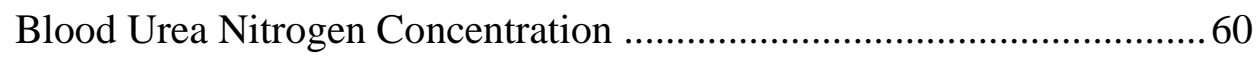

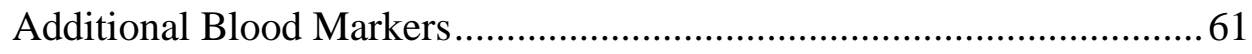

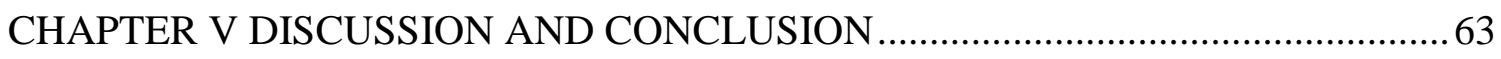

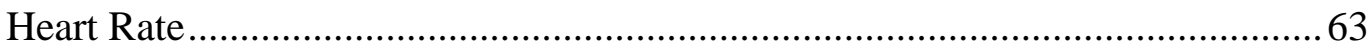

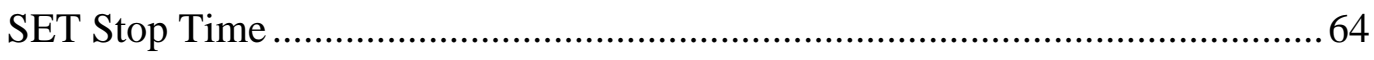

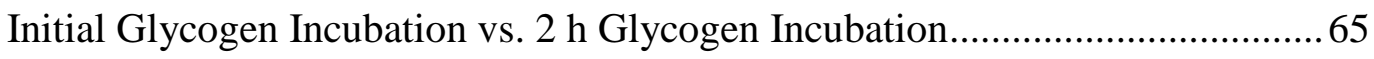

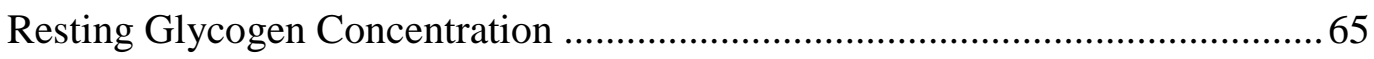

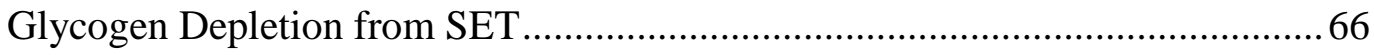

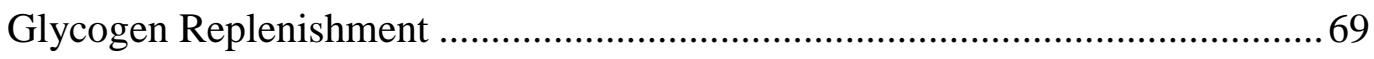

Blood Lactate Concentration............................................................................ 72

Blood Glucose Concentration .........................................................................

Blood Urea Nitrogen Concentration ...................................................................73

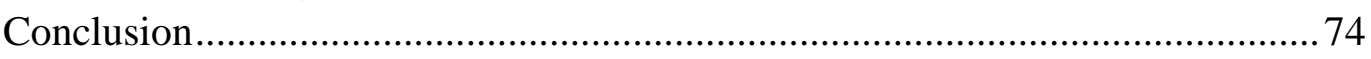

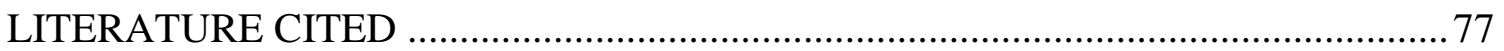

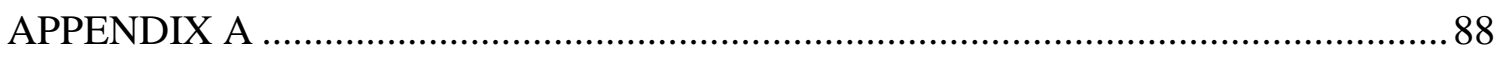

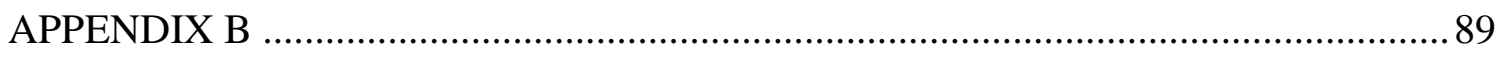

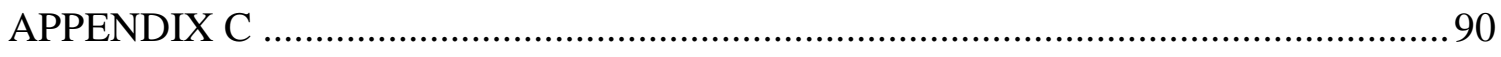

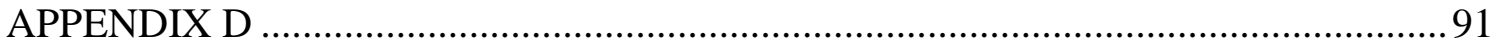

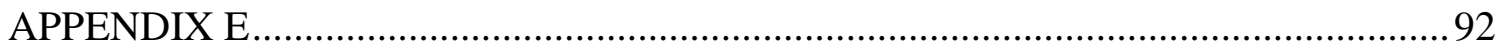

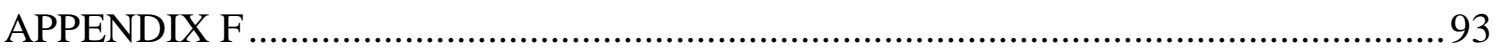

xii 


\section{LIST OF FIGURES}

Page

Figure 1: Biopsy Sample Cut ............................................................................ 43

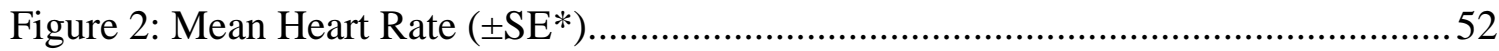

Figure 3: Mean Skeletal Muscle Glycogen Concentration

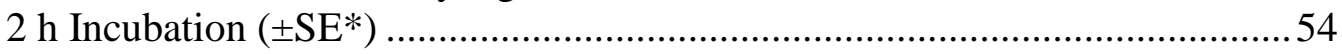

Figure 4: Glycogen Percentage Depletion/Repletion

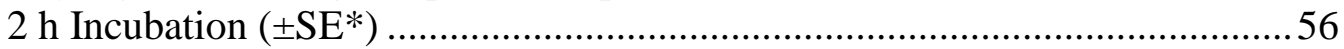

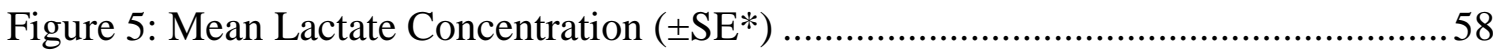

Figure 6: Mean Blood Urea Nitrogen Concentration $\left( \pm \mathrm{SE}^{*}\right)$..................................... 61

Figure 7: Mean Skeletal Muscle Glycogen Concentration

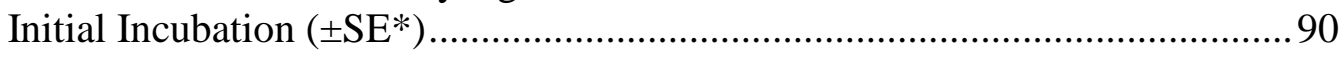

Figure 8: Glycogen Percentage Depletion/Repletion

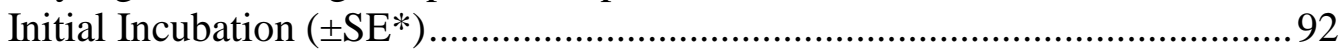




\section{LIST OF TABLES}

Table 1: Starch Concentration of Dietary

Components (100\% DM)

Table 2. Total Analysis of Coastal Bermudagrass

Hay $(100 \%$ DM)

Table 3. Total Analysis of Dietary Treatments

$(100 \%$ DM) 36

Table 4. Experimental Diet Transitions 35

Table 5. Standard Exercise Test (SET) and Muscle Biopsy Groups 39

Table 6. Standardized Exercise Test (SET)

Table 7. Average Body Weight Throughout Project

Table 8. Average Dietary Starch Intake/d, Including Treatment and Hay (As Fed) .........................................................5 50

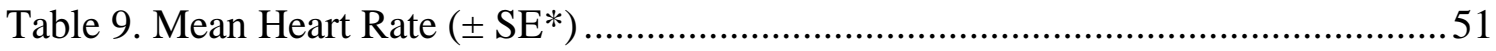

Table 10. Mean Skeletal Muscle Glycogen Concentration ( $\mu \mathrm{g} / \mathrm{mg}$ wet weight) $2 \mathrm{~h}$ Incubation $\left( \pm \mathrm{SE}^{*}\right)$

Table 11. Mean Skeletal Muscle Glycogen Percentage

Depletion/Repletion $2 \mathrm{~h}$ Incubation $\left( \pm \mathrm{SE}^{*}\right)$ 56

Table 12. Mean Blood Lactate Concentration (mmol/L) ( $\left.\pm \mathrm{SE}^{*}\right)$ 58

Table 13. Mean Blood Glucose Concentration (mg/dL) ( $\left.\pm \mathrm{SE}^{*}\right)$ 59

Table 14. Mean Blood Urea Nitrogen Concentration (mg/dL) ( $\left.\pm \mathrm{SE}^{*}\right)$

Table 15. Mean Skeletal Muscle Glycogen Concentration ( $\mu \mathrm{g} / \mathrm{mg}$ wet weight) Initial Incubation $\left( \pm \mathrm{SE}^{*}\right)$

Table 16. Mean Skeletal Muscle Glycogen Percentage Depletion/Repletion Initial Incubation. 


\section{CHAPTER I}

\section{INTRODUCTION}

The prevalence of multi-day competitive events throughout the performance horse industry brings forth the necessity to understand recovery from exercise, especially due to bouts of intense, anaerobic exercise. For example, during a weekend of cutting, a horse may experience several hours of aerobic long trotting during a warm up, followed by anaerobic exercise in the form of preventing the cow from reentering the herd. If the horse scores well enough in the first go, they will return for further go-rounds until the finals. All of this takes place in a period of 24 to $72 \mathrm{~h}$ where horses may become fatigued, resulting in a suboptimal performance during the event finals. This loss of performance may mean loss of award money, notoriety for breeding, or may increase the potential for fatigue-related injuries.

Recently, an industry trend of high fat, high fiber diets has gained popularity and is promoted as a safe way to increase the energy density of a diet. This complicates the issue of fatigue and recovery as anecdotal evidence has shown that horses on a high fat, high fiber, and subsequently low starch, diet may fatigue during multi-day competitions faster than horses on traditional medium or high starch diets. This premature fatigue is likely due to lower resting skeletal muscle glycogen stores in horses fed low starch diets as well as an increased recovery rate before glycogen is replenished for the next exercise period (Kline and Albert, 1981; Waller and Lindinger, 2010).

Muscle glycogen is important fuel for energy during anaerobic metabolism especially during maximal, exhaustive exercise (Harris et al., 1987; Pagan, 1998). A 
reduction in skeletal muscle glycogen stores can result in premature fatigue. Fatigue in athletic competitions is defined as a decrease in performance and the inability to maintain a given exercise intensity (Brooks et al., 2000). Athletes can be observed "hitting a wall" after long term, low intensity exercise with a severe drop in performance due to the near complete depletion of glycogen in muscle fibers (Lindholm et al., 1974; Jentjens and Jeukendrup, 2003). The depletion of glycogen by $41 \%$ does not impair aerobic performance but does effect anaerobic capacity (Topliff, 1984; Lacombe et al., 1999).

While humans and rats can replenish their muscle glycogen stores in as little as 4 to $6 \mathrm{~h}$, replenishment in the horse is 3 to 4 times slower (Jentjens and Jeukendrup, 2003; Waller and Lindinger, 2010). Horses can require between 48 to $72 \mathrm{~h}$ to fully replenish skeletal muscle glycogen stores in both Type I and Type II muscle fibers (Hodgson et al., 1983; Snow and Harris, 1991; Lacombe et al., 2004). Although researchers have successfully increased skeletal muscle glycogen repletion via nutritional intervention in the human, improvements in the horse have been limited (Bergström and Hultman, 1967; Waller and Lindinger, 2010).

Consequently, it is of importance to further explore methods of nutritionally manipulating equine skeletal muscle glycogen replenishment to prevent fatigue and subsequent loss of athletic performance. However, the methods of nutritional intervention must be practical outside of a research setting. In the present study, utilization of commercially available feeds and readily available grains such as steam 
flaked corn are of importance for consumer application. In that way, enhancing glycogen replenishment may be done in a user-friendly manner.

Therefore, the objectives of this study were to compare glycogen concentration in skeletal muscle pre and post exercise and determine subsequent repletion of muscle glycogen in horses fed a high, medium, and low starch diet.

In theory, horses fed a high starch diet should have an increased rate of glycogen replenishment due to substrate availability. Horses fed a high starch diet should have faster glycogen replenishment than medium starch and low starch diets. Utilizing commercially available pelleted rations in conjunction with readily available additives such as corn and alfalfa pellets should allow consumers to readily enhance glycogen replenishment in a field setting. 


\section{CHAPTER II}

\section{LITERATURE REVIEW}

\section{Equine Muscle}

Muscle fibers can be categorized into 2 general classifications of either tonic, red, muscle fibers or tetanic, white, muscle fibers, with red muscles predominantly responsible for posture and white muscle reserved for bursts of work (Beecher et al., 1958; Ashmore et al., 1972). Further classification of muscle fibers are based on fiber size, fiber twitch speed, histochemically by staining, and by energy producing patterns (Ashmore et al., 1972; Essén-Gustavsson and Lindholm, 1985). White muscle fibers are, on average, considerably larger than red muscle fibers (Ashmore et al., 1972). Armstrong and Phelps (1984) observed that in relation to fiber twitch speed, conditions of low force production recruited red muscle fibers while white muscle fibers were progressively recruited with increasing force production, rendering the need for the faster twitch speeds provided by white muscle fibers. This supported a 1972 study by Ashmore et al. which found that red muscle fibers have slower contraction times relative to white muscle fibers. Histochemical stain results vary depending on whether an acidic or alkaline pH is utilized (Brooks et al., 2000; Hodgson et al., 2014). Snow and Guy (1980) reported that at a $\mathrm{pH}$ of 4.3 , dark staining corresponded to red muscle fibers while low to intermediate staining represented white muscle fibers. In reference to energy producing patterns, white muscle fibers exhibit a wide range of succinic dehydrogenase (SDH) and glycogen phosphorylase (GP) activity while red muscle fibers consistently 
exhibited the highest SDH:GP ratio and are therefore adapted for aerobic metabolism (Ashmore et al., 1972).

Most often, equine muscle fiber classification uses the nomenclature as utilized by Brooke and Kaiser (1970), who strove to simplify the nomenclature applied in research. Type I fibers, or slow twitch fibers, are highly oxidative and have a high content of mitochondria which are found in chains between myofibrils, paranuclear, and subsarcolemmal (Beecher et al., 1958; Lindholm and Piehl, 1974; Snow and Guy, 1976). Type I fibers rely on aerobic metabolism for sustained, continuous contractions over an extended period of time and are prevalent in red muscles which contain significantly higher concentrations of myoglobin than white muscles (Beecher et al., 1958; Venes, 2009).

Type II fibers, or fast twitch fibers, are generally glycolytic and contain very low concentrations of mitochondria which are often found in pairs on either side of the $\mathrm{Z}$ line (Snow and Guy, 1976). Type II fibers can be further broken down into Type IIa and Type IIb fibers. Type IIa fibers are also called intermediate fibers and are characterized by possessing greater oxidative capacity than Type IIb fibers (Lindholm and Piehl, 1974; Venes, 2009). The oxidative capacity of Type IIa, along with Type I fibers, is usually high while in Type IIb fibers it can vary markedly (Essén-Gustavsson and Lindholm, 1985). Because of this, Type IIb fibers are often considered the "classic" anaerobic muscle fiber (Brooks et al., 2000; Venes, 2009).

In the human it has been extensively reported that high performing endurance athletes have a greater concentration of Type I fibers, while athletes who excel at 
sprinting have a predominance of Type II fibers (Snow and Guy, 1980). Koni et al. (1977) studied human dizygous and monozygous twins and found that muscle fiber composition was very highly heritable at a rate of $95 \%$. These results have been validated in the horse, with Essén-Gustavsson and Lindholm (1985) reporting Type I to Type II fiber ratios indicated a very strong hereditary influence. Breed to breed differences of muscle fiber type have been reported, with Snow and Guy (1976) finding that Type II fibers in the biceps femoris varied from $82.6 \pm 1.9 \%$ in the Thoroughbred versus $76 \%$ in heavy hunter-type horses. Breeds that are heavier muscled, such as the Quarter Horse, have a higher proportion of Type IIb fibers versus Type IIa fibers (Ashmore et al., 1972). Sigler (1981) reported somewhat higher values in the Quarter Horse at $14.3 \%$ slow twitch and $85.7 \%$ fast twitch in comparison to Snow and Guy's (1976) study.

Training and its effect on muscle fiber characteristics are of great interest in the athletic horse. The fiber types and proportions of a trained Standardbred population (76 $\%$ Type II and $24 \%$ Type I) fibers indicated the well understood notion that horse skeletal muscle is well designed for great endurance and rapid contraction, as is demonstrated in a trotting race (Lindholm and Piehl, 1974). These findings were supported by Essén-Gustavsson and Lindholm's (1985) Standardbred study, in which the focus was solely on training related muscle fiber changes. The subjects were grouped based on time into low, average, and high performing trotters. There were no differences in the proportion of Type I to Type II fibers between the three groups, but the higher performing the group was, the higher the percentage of Type IIa versus Type IIb fibers. 
It was reported that a high oxidative capacity in the muscle and a high Type IIa to Type IIb ratio indicated the highest performing (fastest) Standardbred trotters regardless of sex (Essén-Gustavsson and Lindholm, 1985).

\section{Glycogen in the Equine}

In comparison to the human, the equine has an immense capacity for glycogen storage. Usually, glycogen is present in skeletal muscle in a range of 500 to $650 \mathrm{mmol}$ glycosyl units per kg of dry weight, which is about $50 \%$ higher than what has been reported in humans (Snow and Harris, 1991; Hodgson et al., 2014). Glycogen is often analyzed via skeletal muscle because it is relatively uniform in cellular composition and is the most abundant cellular tissue in the organism (Bergström, 1962). There seems to be no difference between muscle and liver glycogen chains (Bell, 1937).

Equine glycogen is considered a "minimum glycogen molecule" of a chain of 12 glycopyranose units linked by a 1:4- $\alpha$-glycosidic linkages and 2 dimethyl sugar units (Bell, 1937). Similarly to the human, glycogen in the horse is found widely distributed in a granule or rosette formation throughout the sarcoplasm, between the contractile filaments of the myofibrils, and underneath the sarcolemma (Snow and Guy, 1976; Nielsen et al., 2011; Hodgson et al., 2014).

Glycogen is stored primarily in the highly glycolytic white muscle fiber, but is also found in red and intermediate fibers (Baldwin et al., 1973). An example of a white muscle, which is composed of less than $30 \%$ red fibers, is the biceps femoris (Beecher et al., 1958; Armstrong and Phelps, 1984). Although it has been reported that there is small variation in muscle fiber composition within the biceps femoris, this muscle tends 
to have greater concentrations of glycogen compared to other white muscles (Beecher et al., 1958; Snow and Guy, 1980). The biceps femoris is a good candidate for study due to its ease of access, capability of drainage in the event of abscess, relative homogeneity of fiber type, and glycogen content. In the rat, for example, the biceps femoris was found to be composed of $70 \pm 5 \%$ Type IIb fibers, $29 \pm 5 \%$ Type IIa fibers, and $1 \pm 1 \%$ Type I fibers (Armstrong and Phelps, 1984). Snow and Guy (1980) noted that there is a narrower range of fiber composition in the muscle within a particular breed than found in man (Saltin et al., 1977). A heavier muscled breed such as the Quarter Horse would have a higher percentage of Type II fibers and therefore a higher capacity for glycogen storage (Ashmore et al., 1972; Lindholm and Piehl, 1974; Snow and Guy, 1980). Snow and Guy (1980) reported that in the gluteus medius, the Quarter Horse had a significantly greater $(\mathrm{P}<0.05)$ proportion of Type II fibers compared to other breeds.

\section{Metabolism}

The horse has a remarkable capacity for energy production via the 2 main metabolic systems of aerobic and anaerobic metabolism than one might predict through whole body scaling (Harris et al., 1987). Aerobic metabolism requires oxygen and often recruits Type I fibers, thus making this an ideal energy system for slow, long duration work. Aerobic metabolism results in the clean production of ATP and is activated at workloads below $50 \%$ of maximal oxygen uptake capacity (Wasserman and Whipp, 1975; Snow and Mackenzie, 1977). Anaerobic metabolism does not rely on the presence of oxygen, and often activates during fast, short duration work by recruiting Type II fibers. Anaerobic metabolism activates at workloads above $50 \%$ of maximal oxygen 
uptake capacity when metabolism via oxygen cannot produce enough ATP to provide energy for work output (Wasserman and Whipp, 1975). The downside of anaerobic metabolism is the production of lactic acid. Lactic acid is considered a major contributor to fatigue during intense exercise in both man and horse (Tesch, 1980; Nimmo and Snow, 1983; Hodgson et al., 2014). Metabolic systems are not a "one and only" system during an exercise bout, but rather intermingle based on work output.

Glycolysis broadly refers to the process of extracting energy from organic molecules. The energy molecule that makes up the majority of equine diets is carbohydrates (CHO) (Pagan, 1998). Glycolysis is the precursor to both aerobic and anaerobic metabolism. Aerobic metabolism processes $\mathrm{CHO}$ via the production of pyruvate derived from glucose during glycolysis. Anaerobic metabolism processes $\mathrm{CHO}$ via the conversion of 1 molecule of glucose into 2 molecules of lactic acid (Robinson and Harmon, 1941; Holmgren and Ström, 1959; Holloszy, 1967; Snow and Mackenzie, 1977).

During aerobic metabolism the citric acid cycle and oxidative phosphorylation (OXPHOS) are involved in the conversion of glucose into 36 molecules of ATP. Acetylcoenzyme A (acetyl-CoA) is considered the entry molecule for the citric acid cycle and is derived from pyruvate (Brooks et al., 2000). Because the aerobic functional capacity of an individual is dependent on the oxygen transport system, i.e. respiratory function and dimension, it has been of research interest to study the differences between mitochondrial enzyme levels due to training (Larsson et al., 1964; Holloszy, 1967). 
Anaerobic metabolism is of interest in equines because of the extraordinary capacity for lactate accumulation. At resting levels both horse and man have a lactate concentration of $1 \mathrm{mmol} / \mathrm{L}$, but peak lactate levels can reach $30 \mathrm{mmol} / \mathrm{L}$ and $15 \mathrm{mmol} / \mathrm{L}$, respectively (Snow and Mackenzie, 1977; Nimmo and Snow, 1983; Physick-Sheard, 1985). For both horses and humans the lactate threshold is $4 \mathrm{mmol} / \mathrm{L}$ (Wasserman and Whipp, 1975; Brooks et al., 2000; Hodgson et al., 2014).

The primary reason why trained subjects acquire little rise in lactate concentration is due to increases in capillary diffusion per unit of tissue, blood clearance rate, and the muscle to blood gradient (Holloszy, 1967; Harris et al., 1987). This supports the 1941 study by Robinson and Harmon, who noted that with maximal exercise there is no change in basal lactate concentration but rather lactate tolerance. Distance runners performed submaximal work without a significant rise in lactate above basal levels. Although subjects were capable of withstanding greater concentrations of lactate during maximal exercise, an overall decline in lactate concentration during a given workload was noticed. The decline in lactate levels indicated greater circulation to the leg muscles, which distributed lactate faster to allow for whole-body buffering and overall superior oxygen supply to the tissues due to training (Robinson and Harmon, 1941; Holmgren and Ström, 1959; Brooks et al., 2000). A decline in lactate levels when comparing trained and untrained subjects at an identical workload was also observed in the horse (Krzywanek et al., 1976; Snow and Mackenzie, 1977; Physick-Sheard, 1985). However, some dissimilarities between man and horse may be due to differences in changes between lactate dehydrogenase (LDH). Studies in the horse have shown a large 
increase in LDH activity with sprint training, while LDH does not change in sprinttrained men (Thorstensson et al., 1975; Guy and Snow, 1977). However, the horse's more rapid return rate to basal lactate levels is indicative of increased tissue uptake and metabolism due to an increase in oxygen uptake (Robinson and Harmon, 1941;

Holmgren and Ström, 1959; Snow and Mackenzie, 1977; Brooks et al., 2000).

\section{Starch Metabolism in the Equine}

The horse is classified as a hindgut fermenting herbivore (Hurndall, 1896; Hoffman, 2009). Plant cell walls constitute the foundation of any equine diet and are capable of maintaining a mature, sedentary horse (Varloud et al., 2004; NRC, 2007). The need to supplement forage with concentrate grains arises to increase the energy density of feeds (Pagan, 1998; Varloud et al., 2004; Hoffman, 2009). Due to the physiology of the equine digestive system, concentrate supplementation can be detrimental when starch digestion occurs in the hindgut (Kienzle et al., 1992; Kienzle, 1994). Furthermore, equines experience a lower capacity for starch digestion due to a rapid rate of passage of digesta though the foregut and a relatively small and inactive intestinal enzyme population (Kienzle et al., 1997).

The equine foregut is comprised of the esophagus, stomach, and small intestine (SI) and is similar to monogastric animals such as the human and pig. When the horse consumes a concentrate meal, the mastication of feed reduces particle size resulting in a greater surface area. Kienzle (1994) and associates (1997) reported that particle size differed considerably between horses due to individual mastication behaviors and factors such as age and teeth wear. Very little $\alpha$-amylase is present in equine saliva, so limited 
hydrolysis occurs before the bolus arrives in the stomach (Alexander and Chowdhury, 1958; Hoffman, 2009). Once in the stomach, further mixing and particle size reduction occurs and the digesta moves into the SI. Starch digestion is significantly higher in the jejuno-ileum sector of the SI than in the stomach (Varloud et al., 2004). Pancreatic $\alpha$ amylase hydrolyzes non-structural carbohydrates (NSC) with $\alpha-1,4$ linkages (Hoffman, 2009). The 2 classifications of glucose carrier proteins in mammalian cells are high affinity, low capacity, $\mathrm{Na}^{+} /$glucose cotransporter type I (SGLTI) and facilitative glucose transporters (GLUT) (Shirazi-Beechey, 1995; Hoffman, 2009). Sugars accumulate within the enterocytes of the SI brush border and are transported down gradient into systemic circulation (Hoffman, 2009). Compared with other species, pancreatic $\alpha$ amylase activity in the horse is relatively low (Alexander and Chowdhury, 1958; Kienzle, 1994; Varloud et al., 2004; Julliand et al., 2006). In a 2004 study by Varloud et al., starch digestibility was found to be affected by location within the digestive system, the diet, and the interaction between diet and system locale. In conjunction with limited enzymatic activity, the horse has a short transit time in the SI. Because SI starch digestibility decreases with increasing starch intake per meal, the capacity for starch can easily be exceeded (Potter et al., 1992; Kienzle, 1994). This results in starch bypassing enzymatic hydrolysis and entering the hindgut (Potter et al., 1992; Varloud et al., 2004; Julliand et al., 2006; Hoffman, 2009).

The equine hindgut is comprised of the cecum, large intestine (LI), and rectum. Starch that enters the cecum is rapidly fermented by amylolytic bacteria and yields volatile fatty acids (VFAs) such as acetate, propionate, butyrate and to a lesser extent 
lactate (Julliand et al., 2006; Hoffman, 2009). Once starch reaches the LI, starch digestion is almost complete at $91 \pm 6.8 \%$ (Householder, 1978). Variations in this relatively homogenous value vary depending on the microbial population and intestinal environment to which digesta is exposed (Householder, 1978; Julliand et al., 2006).

Although starch can be digested in both the SI and LI, with a total tract digestibility reported by Kienzle (1994) of between $87 \%$ and $100 \%$, it is metabolically more efficient for starch to be metabolized into glucose by enzymatic hydrolysis than by hindgut fermentation into VFAs (Julliand et al., 2006; Hoffman, 2009). Increasing quantities of starch in the diet reduce overall starch digestion due to the concept of starch overload (Potter et al., 1992; Kienzle, 1994). Potter et al. (1992) noticed a tendency for lower starch digestion with higher intake. Due to this, an upper critical limit in SI digestion for meal fed horses was established at 3.5 to $4.0 \mathrm{~g}$ starch $/ \mathrm{kg} \mathrm{BW} \cdot \mathrm{meal}$. Varloud (2004) found that although total tract digestibility was nearly complete in both a fiber based and starch based diet, horses fed high starch had 4 to 6 times more starch reach the hindgut than the fibrous meal (Varloud et al., 2004). Numerous authors also reported values of $4 \%$ to $30 \%$ of bypass starch reaching the hindgut when high starch meals were fed (Householder, 1978; Kienzle et al., 1992; Kienzle, 1994; Rottmann, 1994). Julliand et al. (2006) supported Potter et al. (1992), finding that when the level of starch intake was lower than $200 \mathrm{~g} / 100 \mathrm{~kg} \mathrm{BW} /$ meal, the bypass starch never exceeded 50 $\mathrm{g} / 100 \mathrm{~kg} \mathrm{BW} / \mathrm{meal}$. Above the limit of $200 \mathrm{~g} / 100 \mathrm{~kg} \mathrm{BW} / \mathrm{meal}$, the quantity of starch reaching the hindgut varied from 70 to $150 \mathrm{~g} / 100 \mathrm{~kg} \mathrm{BW} / \mathrm{meal}$ (Julliand et al., 2006). 
Not only is starch reaching the hindgut undesirable in terms of maximizing the energy derived from starch, but dysfunction in the cecum can occur (Kienzle, 1994; Julliand et al., 2001, 2006). A horse fed on forage has higher populations of fibrolytic bacteria in the hindgut. With increasing levels of starch reaching the hindgut, an overgrowth of amylolytic bacteria occurs with a subsequent lysis of the desired bacterial population (Julliand et al., 2001; Hoffman, 2009). Although the fermentation of carbohydrates featuring $\beta-1,4$ linkages generally increases along the length of the digestive tract, fiber digestion decreases in the presence of starch due to the shift in microbial bacteria (Varloud et al., 2004; Julliand et al., 2006; Hoffman, 2009). This bacterium shift creates a more acidic environment in the cecum. A pH of $<6$ favors the production of lactic acid which is associated with clinical conditions such as diarrhea, acidosis, and an increase risk for endotoxemia and laminitis (Julliand et al., 2001; Hoffman, 2009).

While starch digestion is most importantly influenced by the level of intake, other factors affecting starch availability include botanical origin, biochemical structure, and feed processing (Kienzle, 1994; Kienzle et al., 1997; Varloud et al., 2004; Julliand et al., 2006). Starch is the primary component of cereal grains, making up $50 \%$ to $70 \%$ of the grain's dry matter. Corn has the highest starch content of grain commonly fed to horses at $70 \%$, followed by barley (66 \%) and oats (50\%) (Pagan, 1998). The biochemical structure of corn makes digestion limited without processing. Corn contains a very hard meal body with large, firmly fixed granules while oat has a fine hull with single, middle or small sized starch granules (Kienzle et al., 1997). To counteract the 
biochemical characteristics of the grain, processing such as flaking or crushing is utilized to increase the nutritional value of feeds by enhancing the availability of starch. This is done by increasing the surface area of the grain, allowing for greater access to enzymes during digestion.

Mechanical processing, especially grinding, alters the physical form of grain by destroying the macrostructure of connected starch granules and by disturbing the starch granule configuration (Kienzle et al., 1997; Julliand et al., 2006). Interestingly, rolling and crushing (or cracking) of grain does not increase digestibility in relation to whole grains (Kienzle, 1994; Kienzle et al., 1997). This is most likely due to the similarity in particle size of grains processed in this manner in relation to mastication of the horse (Kienzle et al., 1997; Julliand et al., 2006). The most effective form of destroying the crystallinity of corn starch granules is processing with both heat and pressure, such as in steam flaking (Kienzle et al., 1997). Steam flaking leads to the irreversible disruption the starch granule called gelatinization. Highly gelatinized starch granules are often shredded or distorted during processing, thus increasing the surface area and solubility of the granules (Julliand et al., 2006). Although numerous studies have shown that processing grain positively impacts glucose availability and prececal digestion, processing has no effect on fermentation parameters in the hindgut, so it is still of importance to prevent starch overload by monitoring starch intake (Potter et al., 1992; Kienzle et al., 1997; Julliand et al., 2006; Hoffman, 2009). 


\section{Glycogen Depleting Exercise}

Resting glycogen amount varies based on factors such as breed, the muscle being biopsied, and BCS. Davie et al. (1996) reported a resting muscle glycogen concentration of $608 \pm 53.8 \mathrm{mmol} / \mathrm{kg}^{-1}$ glycosyl units in race-trained Thoroughbreds, which supported the resting muscle glycogen concentration of 606 to $667 \mathrm{mmol} / \mathrm{kg}^{-1}$ glycosyl units reported by Snow and Harris (1991). Resting values in the race-trained Standardbred are more varied, with reports of resting muscle glycogen concentration ranging from $472 \pm$ $128 \mathrm{mmol} / \mathrm{kg}$ dry weight, to $622 \pm 150 \mathrm{mmol} / \mathrm{kg}$ dry weight (Lindholm et al., 1974; Hyyppä et al., 1997; Schuback and Essén-Gustavsson, 1998). Similarly to the Standardbred, reported Quarter Horse muscle glycogen concentration has been varied. Miller and Lawrence (1986) reported a resting muscle glycogen concentration of $45.3 \pm$ $2.8 \mathrm{mg} / \mathrm{g}$ wet tissue from the gluteus medius while Sigler (1981) previously reported resting muscle glycogen concentration averaging $17 \mathrm{mg} / \mathrm{g}$ wet tissue from the biceps femoris. The differences in concentrations experienced by the Standardbred and Quarter Horse may be explained by genetic fiber prevalence within various lineages, age, or variations in training programs (Sigler, 1981; Davie et al., 1996; Hodgson et al., 2014).

The BCS system was developed by Henneke et al. (1983) to serve as a universal system to determine the amount of fat cover on a horse and ranges from 1 (extremely emaciated) to 9 (extremely fat). In a 1992 study by Jones and associates, it was reported that control-diet horses in moderate (5) and fat (6) body conditions had significantly $(\mathrm{P}<$ 0.05) greater biceps femoris resting muscle glycogen than horses in thin (3) body condition (Henneke et al., 1983; Jones et al., 1992). Scott et al. (1992) reported 
contradictory results in the same year, with the biceps femoris resting muscle glycogen content of 12 Thoroughbreds scored as 4, 5, or 6 having similar $(\mathrm{P}<0.05)$ glycogen concentrations (Henneke et al., 1983; Scott et al., 1992). These studies are of importance because some performance horses are kept in thinner BCS, such as Thoroughbred racehorses (Hodgson et al., 2014).

It is well documented that during both submaximal and maximal exercise, preferential recruitment of muscle fibers occur in both equine and man, albeit with slight differences (Gollnick et al., 1973b; Lindholm et al., 1974). Both the duration and intensity of an exercise bout is instrumental in muscle fiber recruitment (Lindholm et al., 1974; Hodgson et al., 1983; Hodgson et al., 1985). Lindholm and associates (1974) compared muscle fiber utilization, in terms of exercise intensity, in Standardbreds trotting $300 \mathrm{~m} / \mathrm{min}^{-1}$ (slow trot) for $4 \mathrm{~h}$ or $500 \mathrm{~m} / \mathrm{min}^{-1}$ (fast trot) for $1 \mathrm{~h}$. Using a Periodic Acid-Schiff (PAS) stain it was found that both slow and fast trotting resulted in a selective depletion of skeletal muscle glycogen in the muscle fibers. A preferential depletion pattern of Type I, Type IIa, and Type IIb was observed, although during the fast trotting Type IIa was engaged at the start of exercise which is not observed in man except in the early phase of exercise when the work load is maximal and incapable of being sustained for more than 10 min (Gollnick et al., 1973b).

Hodgson et al. (1983) found the same depletion pattern as increased exercise duration lead to progressive involvement of the faster contracting, more powerful fibers. The same muscle fiber recruitment and glycogen depletion pattern was observed by Snow et al. (1982) in endurance-trained horses completing a $65 \mathrm{~km}$ race. Valberg (1986) 
demonstrated that a graded $\left(6.25^{\circ}\right)$ near maximal treadmill test resulted in the same fiber recruitment pattern. Although depletion was always higher in Type I and the least in Type IIb, variation within Type IIb could be related to the effects of uphill running combined with continually changing demands on the muscle fiber types as the speed constantly increased.

Glycogen depletion and repletion has been studied numerous times in the horse, but research has shown mixed results in the effects of glycogen depletion on fatigue or performance hindrance. Glycogen replenishment in the horse is 3 to 4 times slower than in the human, taking up to $72 \mathrm{~h}$ to replenish versus the human's 4 to $6 \mathrm{~h}$ (Jentjens and Jeukendrup, 2003; Waller and Lindinger, 2010). The rate of glycogen utilization is highly variable due to the type of exercise performed as well as factors such as exercise intensity and diet (Hodgson et al., 1983; Potter et al., 1992; Davie et al., 1994).

Lindholm et al. (1974) demonstrated that glycogen depletion rates varied depending on exercise intensity and differing time points during an exercise bout. Mean glycogen depletion at the slow trot for $4 \mathrm{~h}$ was approximately $21 \mathrm{mmol} / \mathrm{kg} / \mathrm{h}^{-1}$ for the first $3 \mathrm{~h}$ but was sharply reduced to $8 \mathrm{mmol} / \mathrm{kg} / \mathrm{h}^{-1}$ for the last h of exercise. Horses were no longer able to maintain a predetermined pace, even though the Type I fibers were not fully depleted of glycogen and glycogen was still available in Type II fibers. Mean glycogen in the slow trotting horses decreased from a mean of $119 \mathrm{mmol} / \mathrm{kg}^{-1}$ to 48 $\mathrm{mmol} / \mathrm{kg}^{-1}$ and horses fast trotting for $1 \mathrm{~h}$ experienced a skeletal muscle glycogen decline from $103 \mathrm{mmol} / \mathrm{kg}^{-1}$ at rest to 70 and $43 \mathrm{mmol} / \mathrm{kg}^{-1}$ at 30 and $60 \mathrm{~min}$ of exercise, respectively. In this study, skeletal muscle glycogen was utilized 3 times faster per $\mathrm{h}$ 
during a fast trot than during a slow trot, although no glycogen was completely depleted in either exercise regimen (Lindholm et al., 1974).

Hodgson et al. (1983) observed progressive patterns of glycogen depletion and replenishment in horses competing in endurance races. In both $40 \mathrm{~km}$ and $160 \mathrm{~km}$ rides, Type I fibers showed the greatest skeletal muscle glycogen depletion, with the most extensive depletion occurring in horses that completed the $160 \mathrm{~km}$ ride. In these athletes, over $70 \%$ of Type I fibers showed 0 glycogen content post exercise with PAS staining. As the ride distance increased from $40 \mathrm{~km}$ to $160 \mathrm{~km}$, progressive recruitment of the fibers was evident. Repletion occurs in a reverse preferential pattern from Type IIb to I fibers (Lindholm and Piehl, 1974; Snow et al., 1982). In horses that rode $40 \mathrm{~km}$, the Type IIb fibers had similar glycogen content to their pre-ride levels after $24 \mathrm{~h}$. Type I and IIa fibers in the $40 \mathrm{~km}$ horses did not experience complete glycogen replenishment until $48 \mathrm{~h}$ post exercise. In horses that completed the $160 \mathrm{~km}$ ride, repletion was considerably slower with no Type IIb fibers PAS-stained at the 18 to $20 \mathrm{~h}$ post exercise. Even though repletion of glycogen occurs first in Type IIb fibers, the rate of repletion is slow and may not occur in all fibers until $48 \mathrm{~h}$ post exercise. Hodgson et al. (1983) recommended restricting exercise in the post-ride period to allow for maximal repletion of skeletal muscle glycogen stores.

Glycogen depletion in the Thoroughbred is of interest because traditionally they race at maximal levels for short periods of time. Snow and Harris (1991) galloped racetrained Thoroughbreds $1000 \mathrm{~m}$ and $1600 \mathrm{~m}$. There is an obvious requirement for rapid glycogen utilization during these exercise bouts with a previous study reporting 
depletion of $25 \%$ to $33 \%$ (Harris et al., 1987). The gallops resulted in similar glycogen depletion, amounting to approximately $19 \%$ to $25 \%$ of resting concentration. Skeletal muscle glycogen concentration returned to resting values by $72 \mathrm{~h}$. Kitaoka et al. (2014) found that 1 or 2 min of intensive exercise on an inclined $\left(6.0^{\circ}\right)$ treadmill at $120 \%$ $\mathrm{VO}_{2} \max$ (maximal oxygen uptake) also significantly depletes glycogen. The greater levels of depletion in this study may be due to the use of a treadmill and uphill running (Valberg, 1986; Kitaoka et al., 2014). Davie et al. (1999) also looked at glycogen depletion of Thoroughbreds during a submaximal treadmill test. Horses in protocol A completed a low intensity exercise test to deplete primarily Type I fibers, while horses in protocol B performed short burst of high intensity exercise to primarily deplete Type II fibers, and the control horses did not perform any exercise before the SET. Five $h$ after each exercise protocol, all horses completed a SET at $60 \%$ of their $\mathrm{VO}_{2}$ max. While there was no significant difference between skeletal muscle glycogen concentration before the protocols, there was a significant decrease in concentration in protocols $\mathrm{A}$ and $\mathrm{B}$ in relation to the control group after the protocols were performed. However, there was no significant difference between protocols A and B with mean glycogen depletion of $21 \%$ and $29 \%$, respectively. The highest individual rate of glycogen depletion was $58 \%$ following protocol B.

Topliff (1984) reported a decrease in the relative work capacity in horses pulling sleds when glycogen was depleted by $41 \%$. Lacombe et al. (1999) supported this by concluding that after $3 \mathrm{~d}$ of strenuous exercise a depletion in skeletal muscle glycogen concentration by $55 \%$ does not impair aerobic performance but does effect anaerobic 
capacity in horses. Davie and associates' 1996 Thoroughbred study had contrasting results to these works. This study demonstrated that a skeletal muscle glycogen depletion of $22 \%$ can occur without having a significant effect on work capacity during high intensity exercise. The argument against this study is that the glycogen depletion that occurred was not reduced sufficiently to provide measurable effects on anaerobic performance (Lacombe et al., 1999).

Due to the lengthy amount of time it takes for glycogen to replenish to pre exercise values in the horse, it is of interest to conduct research involving multiple $\mathrm{d}$ of exercise. Hyyppä et al. (1997) conducted a study on Standardbreds over the course of 3 d. Horses completed 2 trials, with trial A consisting of trotting 3000 m, 3000 m, and $2000 \mathrm{~m}$ followed by trial B $3 \mathrm{~d}$ later consisting of trotting $2100 \mathrm{~m}, 2100 \mathrm{~m}$, and $1600 \mathrm{~m}$. Skeletal muscle glycogen concentration after the $2000 \mathrm{~m}$ and $1600 \mathrm{~m}$ trots of trials A and B was significantly lower than pre-trial values. Interestingly, a further decrease in glycogen concentration occurred $4 \mathrm{~h}$ post trial. For trial A, skeletal muscle glycogen was depleted by averages of $30.44 \%$ immediately post trial, $50.74 \% 4 \mathrm{~h}$ post trial with repletion occurring at $4.72 \%$ at $24 \mathrm{~h}$ post trial, and $46.78 \%$ at $48 \mathrm{~h}$ post trial. For trial B, skeletal muscle glycogen was depleted by averages of $26.48 \%$ immediately post trial, $43.43 \% 4 \mathrm{~h}$ post trial with repletion occurring at $1.87 \%$ at $24 \mathrm{~h}$ post trial, $48.69 \% 48 \mathrm{~h}$ post trial, and a depletion of $29.72 \%$ at $72 \mathrm{~h}$ post trial. A similar decrease in glycogen concentration after $24 \mathrm{~h}$ during recovery has been reported (Vonderohe, 2013). The absence of glycogen replenishment during the first $4 \mathrm{~h}$ of recovery is consistent with earlier studies in horses, but contradicts Schuback and Essén-Gustavsson (1998), who 
reported glycogen repletion occurring during the first 15 min post exercise (Snow et al., 1982; Valberg, 1986; Davie et al., 1995). However, immediate glycogen replenishment could be explained by the conversion of lactate and glucose-6-phosphate produced during intense exercise back into glycogen (Harris et al., 1987; Schuback and EssénGustavsson, 1998). This instantaneous glycogen replenishment after exercise is also consistent with studies in humans and rats (Bergström and Hultman, 1966; Fell et al., 1980; Casey et al., 1995). Bonen et al. (1985) indicated that in humans mild exercise delays the onset of glycogen replenishment during a recovery period. Because the pattern of glycogen repletion up to $48 \mathrm{~h}$ post exercise was similar for trial A and B, the dominant factor in the rate of glycogen replenishment would be the repetition of exercise rather than exercise intensity (Hyyppä et al., 1997).

Fatigue in athletic competition is defined as a decrease in performance and the inability to maintain a given exercise intensity (Brooks et al., 2000). Fatigue is a complex sequence of events that may result from multiple factors dependent on exercise. Although extensive glycogen depletion would inevitably limit muscular work, it would be unlikely to be the sole cause of fatigue in a multitude of studies as muscle fibers were not completely devoid of glycogen post exercise (Lindholm and Saltin, 1974; Hodgson et al., 1985; Miller and Lawrence, 1986).

Lindholm et al. (1974) determined that fatigue was due to metabolic factors and muscle fiber utilization even though no fibers were completely depleted of glycogen. Hodgson et al. (1985) had similar results in that glycogen was present in all muscle fibers post exercise. Other causes that may have contributed to fatigue in eventing 
include metabolic acidosis resulting from lactate accumulation, altered electromotor activity, depletion of creatine phosphate or the factors involved in its resynthesis, and alterations in the potassium gradient between intra and extracellular locations of muscle tissue (Saltin et al., 1977; Hodgson et al., 1985; Brooks et al., 2000).

Metabolic acidosis occurs when lactic acid production exceeds its disposal and clearance (Brooks et al., 2000). Lactate dissociates a hydrogen proton $\left(\mathrm{H}^{+}\right)$, which can lower $\mathrm{pH}$. Within the muscle, a lower $\mathrm{pH}$ may inhibit phosphofructokinase (PFK) and thus inhibit glycolysis (Spencer et al., 1992; Brooks et al., 2000; Hodgson et al., 2014). A high correlation between the percentage of Type II fibers and lactic acid production exists, as these fibers are responsible for anaerobic glycolysis (Valberg, 1986). During flat racing, lactate can accumulate to up to $30 \mathrm{mmol} / \mathrm{L}$ in the Thoroughbred (PhysickSheard, 1985). The resulting metabolic acidosis is thought to be more likely to be a primary cause of fatigue than the depletion of muscle glycogen.

Other factors that may contribute to fatigue include skeletal muscle glycogen concentration before exercise and ammonia accumulation. Not only could low concentrations of glycogen pre exercise affect subsequent athletic performance, but low glycogen content in muscles results in an increased rate of protein degradation during exercise (Gollnick et al., 1973a; Lemon, 1987). Increased protein degradation could lead to the onset of overtraining and stress (Hyyppä et al., 1997; Kreider et al., 1997; McGowan and Whitworth, 2008). The effects of ammonia on muscle metabolites has been well studied in man, but has been overlooked as a cause of fatigue in the horse (Gollnick et al., 1973a; Miller and Lawrence, 1986; Greenhaff et al., 1991). Gorostiaga 
et al. (2014) measured blood ammonia as a marker of muscle metabolites in men during leg press exercises. A high correlation $(\mathrm{r}=0.90, \mathrm{P}<0.01)$ was found between muscle and blood lactate values as well as a significant negative correlation between blood ammonia concentrations and ATP content in the muscle $(\mathrm{r}=-0.75, \mathrm{P}<0.01)$. Because ATP is required for both aerobic and anaerobic metabolism, ATP can easily be considered the limiting factor in terms of fatigue (Brooks et al., 2000; Gorostiaga et al., 2014; Hodgson et al., 2014).

\section{Human Nutritional Intervention}

It was first reported in the late 1960's that the ingestion of carbohydrates after exercise promoted the replenishment of skeletal muscle glycogen (Bergström and Hultman, 1967; Carrithers et al., 2000). Since then, there has been success in humans in terms of enhancing glycogen replenishment though nutritional intervention. An important goal of any athlete's diet is to provide muscles with the needed substrates to fuel performance and optimize the restoration of glycogen between workouts (Burke et al., 2004). With this being said, post exercise nutritional enhancements have been of interest in terms of $\mathrm{CHO}$ supplementation, protein supplementation, and high fat diets in the human athlete.

Bergström and Hultman (1966) originally found that a CHO-rich diet increased muscle glycogen content far above resting values when comparing a leg exercised via a cycle ergometer to the alternate resting leg. Subjects consumed diets high in $\mathrm{CHO}$ for 2 $\mathrm{d}$ following exercise. One d post exercise glycogen concentration in the exercised leg increased from nearly entirely depleted to values higher than the skeletal muscle 
concentration of the rested leg. The glycogen content continued to rise in the exercised leg following $2 \mathrm{~d}$ of the $\mathrm{CHO}$-rich diet, with glycogen concentration in the exercised leg approximately twice as high as glycogen concentration in the rested leg. From this experiment, an increase in research occurred to understand the mechanisms behind $\mathrm{CHO}$ loading and the optimum level of CHO post exercise (Jentjens and Jeukendrup, 2003; Burke et al., 2004).

Ivy et al. (1988a) set out to determine if the rate of glycogen replenishment could be increased during a period of $4 \mathrm{~h}$ post exercise based on the amount of $\mathrm{CHO}$ consumed. Although skeletal muscle glycogen storage was significantly increased in subjects that consumed 2 varying levels of glucose supplement, there was no difference between the different glucose treatments. These findings were supported by another study which found that a $25 \%$ glucose polymer supplement given at $2.0 \mathrm{~g} / \mathrm{kg} \mathrm{BW}$ caused an increase in glycogen storage of $300 \%$ above basal levels during $2 \mathrm{~h}$ of recovery and that delaying ingestion of a glucose polymer by $2 \mathrm{~h}$ resulted in a $47 \%$ slower rate of glycogen storage Ivy et al. (1988b).

Zawadzki et al. (1992) reported that feeding $112 \mathrm{~g} \mathrm{CHO}$ with $40.7 \mathrm{~g}$ protein resulted in significantly faster glycogen storage than a diet of all $\mathrm{CHO}$ or all protein. Van Loon et al. (2000) supported these results with findings of feeding amino acids to a CHO solution at an intake of $0.8 \mathrm{~g} \mathrm{CHO} / \mathrm{kg}^{-1} / \mathrm{h}^{-1}$ can stimulate glycogen synthesis. However, glycogen synthesis can be accelerated by feeding a total of $1.2 \mathrm{~g} / \mathrm{kg}$ at $30 \mathrm{~min}$ intervals. Later studies supported feeding a CHO-based diet maintains a maximal rate of glycogen 
storage for as many as 4 to $6 \mathrm{~h}$ after exercise (Van Loon et al., 2000; Jentjens and Jeukendrup, 2003).

Fat supplementation has shown to have a glycogen sparing effect by decreasing resting glycogen stores and increasing time to fatigue in both humans and rats (Conlee et al., 1990; Saitoh et al., 1994; Jentjens and Jeukendrup, 2003). Subjects that CHO loaded when previously fed a high fat diet experienced increased rates of muscle glycogen repletion than subjects previously fed a CHO diet (Saitoh et al., 1994). Part of the adaptation to feeding fat may involve increased storage and mobilization of triglycerides (TG) (Conlee et al., 1990). Fat adaptation allows for glycogen sparing, as muscle cells preferentially utilize TG during aerobic metabolism, thus "sparing” glycogen stores for use during anaerobic metabolism (Jentjens and Jeukendrup, 2003).

\section{Equine Nutritional Intervention}

Manipulation of equine diets to promote athletic performance has been of importance especially due to the success of supercompensation of skeletal muscle glycogen in the human athlete as well as successes in fat adaptation in humans, rats, and dogs (Bergström and Hultman, 1966; Conlee et al., 1990; Reynolds et al., 1997; Burke et al., 2004; Geor, 2007). Manipulation via diets higher in CHO, fat supplementation, and other means of supplementation have been the primary focus of equine studies in the dietary manipulation of glycogen replenishment.

Kline and Albert (1981) were the first to report the effects of CHO diet manipulation. They found that enhancing muscle glycogen stores in Standardbred horses via a program of glycogen "loading" was effective $(\mathrm{P}<0.01)$ to a small extent, but not 
nearly as effective in human studies (Bergström et al., 1967; Gollnick et al., 1973a). Topliff (1984) found that horses fed a high CHO diet had approximately a $36 \%$ higher skeletal muscle glycogen concentration compared to horses on a high protein, high fat diet. This study expanded on Kline and Albert's (1981) work with an increase in the length of time horses were on the dietary treatment. Interestingly, both investigators had similar results with various adaptation periods, showing that horses are capable of displaying a significant glycogen response in as little as $3 \mathrm{~d}$ (Kline and Albert, 1981). Lacombe et al. (2004) supported both of these findings by reporting that rates of glycogen synthesis was higher when horses were fed a diet containing $50.9 \%$ starch versus diets of $22.2 \%$ and $4.3 \%$ starch. Vonderohe (2013) found that horses require greater than $553.7 \mathrm{~g}$ starch/d for optimum recovery from exercise depleting both Type I and Type II fibers. Dietary starch is significant in glycogen replenishment, as a low starch diet had a slower overall rate of glycogen replenishment until at least $48 \mathrm{~h}$ post exercise.

Due to the finding that glycogen replenishment in the horse can take multiple days, it is of interest to look at studies observing glycogen replenishment over multiple days of glycogen depleting exercise (Waller and Lindinger, 2010). Interestingly, JoseCunilleras et al. (2006) found that horses fed 2 high $\mathrm{CHO}$ meals in the $8 \mathrm{~h}$ following the 3rd day of consecutive exercise had 3 times greater whole-body glucose kinetics, but only marginally affected muscle glycogen concentrations. At $24 \mathrm{~h}$ post exercise, horses fed high $\mathrm{CHO}$ had higher skeletal muscle glycogen concentrations than their pre exercise values $(\mathrm{P}=0.04)$. More recently Pagan et al. (2015) conducted a 3 d depletion study 
with HS, MS, and LS diets consisting of $65.2 \%, 46.5 \%$, and $12.4 \%$ NSC. At 72 h skeletal muscle glycogen replenished $94 \%, 94 \%$, and $63 \%$ for HS, MS, and LS, correspondingly. Low NSC diets do not seem to provide enough substrate to significantly $(\mathrm{P}<0.05)$ replenish glycogen stores at $72 \mathrm{~h}$.

Fat supplementation is thought to increase lipoprotein lipase allowing for the horse to have increased capacity for oxidation of fatty acids (FA) in the muscle (Geor, 2007). This "spares" glycogen stores by utilizing FA during aerobic metabolism and saving skeletal muscle glycogen for anaerobic metabolism (Jentjens and Jeukendrup, 2003). The efficacy of fat supplementation is debatable, as authors have reported both improved performance during low intensity exercise and no changes due to supplementation (Jose-Cunilleras and Hinchcliff, 2004; Geor, 2007).

Hambleton et al. (1981) reported a threshold of $16 \%$ fat in the diet for effective fat supplementation. Feeding fat at levels ranging from $4 \%$ to $16 \%$ served as a safe way increase the energy density of a feed. However when feeding $16 \%$ fat, resting skeletal muscle glycogen values were lower than other diets. When feeding $12 \%$ fat, increased dietary fat resulted in a $46 \%$ increase in resting glycogen concentrations. Pagan et al. (1987) reported that although the respiratory quotient (RQ) for submaximal exercise was lower in a high fat diet, results did not support the hypothesis that muscle glycogen was spared. Meyers et al. (1989) found that fat supplementation reduced the amount of concentrate needed to maintain body condition. Meyers et al. (1989) and Oldham et al. (1990) both concluded that fat supplementation increased skeletal muscle glycogen concentrations as well as enhanced performance past the anaerobic threshold. 
Jones et al. (1992) and Scott et al. (1992) both reported on the efficacy of fat supplementation. Horses adapted to fat supplemented diets had increases in skeletal muscle glycogen stores pre-exercise, which agreed with previous research (Hambleton et al., 1981; Meyers et al., 1989; Oldham et al., 1990). When Scott et al. (1992) supplemented fat to Thoroughbreds while altering BCS up and down, all body conditions significantly $(\mathrm{P}<0.05)$ increased in skeletal muscle glycogen concentrations by between $30 \%$ and $55 \%$. Horses also mobilized greater amounts of glycogen than the control diet $(\mathrm{P}<0.05)$, thus allowing greater amounts of muscle glycogen for glycogenolysis and glycolysis during subsequent anaerobic work (Scott et al., 1992; Geor, 2007).

Alternative methods of nutritional intervention include administering treatments via nasogastric tube (NG) or I.V. Davie et al. (1994) found that administering $3 \mathrm{~g} / \mathrm{kg}^{-1}$ NG after exercise had no effect on the rate of glycogen synthesis. Another study lead by Davie found that the rate of glycogen resynthesis during the first $12 \mathrm{~h}$ after exercise was greatly enhanced by administering $6 \mathrm{~g} / \mathrm{kg}^{-1}$ dextrose I.V. as a $20 \%$ solution in an isotonic electrolyte solution (Davie et al., 1995). Waller et al. (2009) found that NG administration of acetate resulted in an enhanced rate of muscle glycogen replenishment during the initial $4 \mathrm{~h}$ post exercise. By $24 \mathrm{~h}$, there was no difference between acetate treated horses and the control group. Waller et al. (2009b) also found that NG supplementation with an electrolyte solution enhanced the rate of muscle glycogen resynthesis and resulted in a faster restoration of hydration post exercise. Although there has been success with NG or I.V. supplementation, the amount of solution required to be 
beneficial as well as the feasibility for administration outside of a research setting need to be considered. 


\section{CHAPTER III}

\section{MATERIALS AND METHODS}

\section{Animal Management}

American Quarter Horse mares $(n=5)$ and geldings $(n=4)$ owned by the Texas

A\&M University Department of Animal Science were used in a 3 x 3 Latin square with replication study lasting $105 \mathrm{~d}$. Horses were from 2 to $7 \mathrm{yr}$ of age (average age $4.5 \mathrm{yr}$ ) with an average BW of $463 \mathrm{~kg}$ and were selected based on temperament, health, and soundness. The horses were vaccinated and dewormed prior to the study according to a regular schedule maintained by the Texas A\&M Horse Center. Horses were dewormed halfway through the project as per the regular farm schedule. Horses' hooves were trimmed prior to the study and were trimmed $7 \mathrm{~d}$ prior to each SET. Horses were individually housed at the Texas A\&M Horse Center in pens measuring $14.17 \mathrm{~m}$ by $11.88 \mathrm{~m}$ and were managed in accordance with the guidelines established by the Institutional Animal Care and Use Committee.

The study consisted of three $14 \mathrm{~d}$ experimental periods during which the horses were fed 1 of 3 concentrates, one $7 \mathrm{~d}$ washout period where horses were fed 1 concentrate, and three $7 \mathrm{~d}$ transition periods during which horses were gradually acclimated to a different diet. Horses were fed comparable Coastal Bermudagrass (Cynodon dactylon) (hay A) for $7 \mathrm{~d}$ during the beginning of the study and were then switched to a batch of Coastal Bermudagrass (hay B) for the remaining $98 \mathrm{~d}$ of the experiment. Concentrate diets were formulated in an isocaloric manner with the only 
significant variable the starch concentration of the diet. Horses were weighed weekly to maintain consistent feeding protocol.

During the first experimental diet period, 1 gelding that was receiving the medium starch (MS) diet developed diarrhea. Upon evaluation by a Texas A\&M Large Animal Field Service veterinarian, it was determined that the gelding had colitis which was unrelated to the diet, and was removed from the project. No other horses exhibited diarrhea. The gelding was removed and replaced with a younger gelding. This gelding followed the same schedule but was one biopsy behind i.e., his first muscle biopsy occurred when the other 8 horses received their second muscle biopsy, etc.

\section{Dietary Treatment}

Horses were fed 1 of 3 diets of either a high starch (HS) diet with $56 \%$ commercial pelleted ration A and $44 \%$ steam flaked corn (SFC), a medium starch (MS) diet with only commercial pelleted ration A, or a low starch (LS) diet with $70 \%$ commercial pelleted ration $\mathrm{B}, 30 \%$ alfalfa pellets (AP), and added corn oil (CO) to meet remaining energy requirements (average $60 \mathrm{~g}$ ). Horses received $1 \% \mathrm{BW}$ (as fed) of Coastal Bermudagrass hay daily. Horses were fed to light working horse requirements from the Nutrient Requirements of Horses: Sixth Revised Edition (NRC, 2007). Daily calories not met through Coastal Bermudagrass hay were met with a concentrate treatment diet that averaged $3.293 \mathrm{~kg} / \mathrm{d}$. Daily hay and concentrate rations were divided equally into 2 meals, fed at $0700 \mathrm{~h}$ and $1900 \mathrm{~h}$. Hay and concentrate were offered to each horse in an individual elevated feeder. Horses had individual water troughs or buckets to monitor intake and had ad libitum access to water throughout the study. Refusals at the 
time of the next feeding were collected and weighed. Total analysis of each concentrate and hay are presented in Tables 1,2, and 3.

Prior to each $14 \mathrm{~d}$ experimental diet period, horses were gradually acclimated to the new diet over a transition period of $7 \mathrm{~d}$. The first $3 \mathrm{~d}$ ( 6 feedings) horses were fed 25 $\%$ of the new diet (i.e., LS mix) and $75 \%$ of the previous diet (i.e., HS mix). The next 2 $\mathrm{d}$ (4 feedings) were presented at $50 \%$ new diet and $50 \%$ previous diet. The final $2 \mathrm{~d}(4$ feedings) consisted of $75 \%$ new diet and $25 \%$ of the previous diet. Horses were randomly placed into 3 groups with $n=3$ per group and were assigned 1 of the 3 experimental diets. The MS feed was used as a washout feed for $7 \mathrm{~d}$ prior to the first transition period. The MS diet was selected because horses were on a comparatively similar diet before the start of the study. Horses were transitioned from HS to LS to MS (Table 4) throughout the course of the project to prevent any adverse reactions to starch amount. Transition periods occurred in a staggered manner because there were 3 collection days. This ensured that all horses were on each diet for $14 \mathrm{~d}$. 
Table 1. Starch Concentration of Dietary Components (100\% DM)

\begin{tabular}{l|c}
\hline \multicolumn{1}{c}{ Component } & Starch Concentration, \% \\
\hline High Starch Mix $^{\mathrm{a}}$ & 39.30 \\
\hline Medium Starch Concentrate $^{\mathrm{a}}$ & 24.80 \\
\hline Low Starch Mix $^{\mathrm{b}}$ & 6.20 \\
\hline Coastal Bermudagrass Ac & 2.30 \\
\hline Coastal Bermudagrass B & 1.50 \\
\hline${ }^{\mathrm{a}}$ Commercial Strategy GX (Purina Animal Nutrition LLC) and SFC (Producers \\
Cooperative Association, Bryan, TX) \\
${ }^{\mathrm{a}}$ Commercial Strategy GX (Purina Animal Nutrition LLC) \\
${ }^{\mathrm{b}}$ Commercial Strategy Healthy Edge (Purina Animal Nutrition LLC), AP (Producers \\
Cooperative Association, Bryan, TX), and CO (ACH Food Companies, Inc.) \\
${ }^{\mathrm{c}}$ Coastal Bermudagrass fed for 98 d of study \\
${ }^{\mathrm{d}}$ Coastal Bermudagrass fed for 7 d of study
\end{tabular}


Table 2. Total Analysis of Coastal Bermudagrass Hay (100 \% DM)

\begin{tabular}{|l|c|c|}
\hline \multicolumn{1}{c|}{ Component } & \multicolumn{2}{c}{ Hay Sample } \\
\hline Moisture, \% & A & B \\
\hline Crude Protein, \% & 5.6 & 6.5 \\
\hline Acid Detergent Fiber (ADF), \% & 8.5 & 11.5 \\
\hline Neutral Detergent Fiber (NDF), \% & 37.1 & 38.7 \\
\hline Non-Fiber Carbohydrates (NFC), \% & 67.2 & 70.9 \\
\hline Starch, \% & 14.3 & 7.6 \\
\hline Total Dietary Nutrients (TDN), \% & 2.3 & 0.8 \\
\hline Calcium, \% & 55 & 54 \\
\hline Phosphorus, \% & 0.55 & 0.36 \\
\hline Magnesium, \% & 0.31 & 0.20 \\
\hline Potassium, \% & 0.13 & 0.14 \\
\hline Sodium, \% & 1.99 & 0.69 \\
\hline Dietary Energy (DE), Mcal/kg & 0.061 & 0.800 \\
\hline
\end{tabular}


Table 3. Total Analysis of Dietary Treatments (100\% DM)

\begin{tabular}{|l|c|c|c|}
\hline \multicolumn{1}{|c}{ Component } & \multicolumn{3}{c|}{$\begin{array}{c}\text { Treatment } \\
\text { MS }^{\mathbf{b}}\end{array}$} \\
\hline Moisture, \% & HS $^{\mathbf{a}}$ & 9.5 & 5.7 \\
\hline Crude Protein, \% & 10.6 & 16.2 & 15.7 \\
\hline Acid Detergent Fiber (ADF), \% & 14.2 & 14.4 & 25.9 \\
\hline $\begin{array}{l}\text { Neutral Detergent Fiber (NDF), } \\
\%\end{array}$ & 9.5 & 31.7 & 42.8 \\
\hline Starch, \% & 21.8 & & \\
\hline Crude Fat (Acid Hydrolysis), \% & 39.3 & 24.8 & 6.2 \\
\hline Total Dietary Nutrients (TDN), \% & 6.9 & 9 & 13.6 \\
\hline Calcium, \% & 80 & 77 & 74 \\
\hline Phosphorus, \% & 1.02 & 1.47 & 1.31 \\
\hline Magnesium, \% & 0.56 & 0.75 & 0.41 \\
\hline Potassium, \% & 0.23 & 0.31 & 0.22 \\
\hline Sodium, \% & 0.79 & 1.10 & 1.74 \\
\hline Dietary Energy (DE), Mcal/kg & 0.18 & 0.25 & 0.25 \\
\hline
\end{tabular}

${ }^{a}$ Commercial Strategy GX (Purina Animal Nutrition LLC) and SFC (Producers Cooperative Association, Bryan, TX)

${ }^{\mathrm{b}}$ Commercial Strategy GX (Purina Animal Nutrition LLC)

${ }^{\mathrm{c}}$ Commercial Strategy Healthy Edge (Purina Animal Nutrition LLC), AP (Producers

Cooperative Association, Bryan, TX), and CO (ACH Food Companies, Inc.) 
Table 4. Experimental Diet Transitions

\begin{tabular}{|c|c|c|c|}
\hline & Group 1 & Group 2 & Group 3 \\
\hline & \multicolumn{3}{|c|}{$\mathrm{n}=$ mares $;$ geldings } \\
\hline & $\mathrm{n}=1 ; 2$ & $\mathrm{n}=2 ; 1$ & $\mathrm{n}=2 ; 1$ \\
\hline 7-d Washout Period & MS & MS & MS \\
\hline 7-d Transition Period & MS to HS & MS to LS & MS to MS \\
\hline 14-d Diet Period & HS & LS & MS \\
\hline \multicolumn{4}{|l|}{ Treadmill/Muscle Biopsy } \\
\hline 7-d Transition Period & HS to LS & LS to MS & MS to HS \\
\hline 14-d Diet Period & $\mathrm{LS}$ & MS & HS \\
\hline \multicolumn{4}{|l|}{ Treadmill/Muscle Biopsy } \\
\hline 7-d Transition Period & $\mathrm{LS}$ to $\mathrm{MS}$ & MS to HS & HS to LS \\
\hline 14-d Diet Period & MS & HS & LS \\
\hline Treadmill/Muscle Biopsy & & & \\
\hline
\end{tabular}

\section{Exercise Protocol}

Prior to the trial, all 9 horses had previously been used in Texas A\&M University Department of Animal Science horse training labs. Horses were exercised throughout the trial at a walk and trot in a 6-slot panel exerciser (Freestyle Equine Equipment, Unadilla, GA). Horses were exercised $3 \mathrm{~d} /$ wk for 30 min at $40 \%$ walk and $60 \%$ trot to properly simulate light exercise to maintain, but not increase, fitness (NRC, 2007). One horse per exercise group would wear a heart rate monitor (Polar Electro, Lake Success, NY) to ensure that the average target heart rate of $80 \mathrm{bpm}$ was reached. Horses travelled an average of $5.8 \mathrm{~km}$ per exercise session at an average speed of $4.1 \mathrm{~m} / \mathrm{s}$. 
Horses were placed into 2 exercise groups $(n=5 ; n=4)$ based on their housing order to prevent fighting during exercise. Horses were exercised at 0900 and 1000 on exercise days. Horses were exercised on a forward (clockwise) panel cycle for the 0900 exercise bout and on the reverse (counter clockwise) panel cycle for the 1000 exercise bout. Exercise groups alternated the first or second exercise bout every exercise day to negate any differences between ambient temperature and direction of the panel cycle. For example, 1 exercise group would work in the forward direction at 0900 for the first exercise day and would switch and work in the reverse direction at 1000 for the next exercise day. When horses were not being worked they were turned out in their designated individual pen.

Horses were grouped into 3 separate groups on SET days. This was done due to the time required for the SET and biopsy procedures and the amount of horses in the study. One horse per diet was in each group, with a mix of mares and geldings as displayed in Table 5. Horses were rested an average of $4 \mathrm{~d}$ prior to the SET. After the SET, horses were rested for an average of $8 \mathrm{~d}$ before resuming the light exercise schedule. 
Table 5. Standard Exercise Test (SET) and Muscle Biopsy Groups

\begin{tabular}{|c|c|c|c|}
\hline & $\begin{array}{c}\text { Rest d Pre } \\
\text { SET }\end{array}$ & $\begin{array}{c}\text { Rest d Post } \\
\text { SET }\end{array}$ & $\begin{array}{c}\text { Muscle Biopsy Groups } \\
\text { (mares; geldings) }\end{array}$ \\
\hline 1st Day of Collections & 5 & 9 & $\mathrm{n}=2, \mathrm{n}=1$ \\
\hline 2nd Day of Collections & 4 & 8 & $\mathrm{n}=2, \mathrm{n}=1$ \\
\hline 3rd Day of Collections & 3 & 7 & $\mathrm{n}=1, \mathrm{n}=2$ \\
\hline Averages & 4 & 8 & \\
\hline
\end{tabular}

After each $14 \mathrm{~d}$ experimental diet period each horse performed a SET (Table 6). Horses were first worked for $30 \mathrm{~min}$ at a brisk trot $(4.1 \mathrm{~m} / \mathrm{s})$ in a panel exerciser and immediately moved to a high speed treadmill (Sato, Upsala, Sweden) to be worked to fatigue. Fatigue was considered met when the HR remained above $180 \mathrm{bpm}$ for more than $30 \mathrm{~s}$, the horse completed the full $27 \mathrm{~min}$ SET, or the horse could no longer keep pace with the treadmill, meaning the horse could no longer safely complete the SET. Heart rates were measured with the same monitors used during the exercise regimen to maintain fitness. Intensity of the exercise was increased in an incremental fashion with alternating increases between speed and incline of the treadmill. The total distance travelled in the panel exerciser and on the high speed treadmill was 7,380 $\mathrm{m}$ and 7,098 $\mathrm{m}$, respectively. 
Table 6. Standardized Exercise Test (SET)

\begin{tabular}{|l|l|l|}
\hline \multicolumn{1}{|c|}{ Time (min) } & \multicolumn{1}{c|}{ Speed $(\mathbf{m} / \mathbf{s})$} & \\
\hline $30^{\mathrm{a}}$ & 4.1 & $0.0^{0}$ \\
\hline $2^{\mathrm{b}}$ & 1.9 & $0.0^{0}$ \\
\hline $5^{\mathrm{b}}$ & 3.0 & $0.0^{0}$ \\
\hline $5^{\mathrm{b}}$ & 3.0 & $2.1^{0}$ \\
\hline $5^{\mathrm{b}}$ & 5.2 & $2.1^{0}$ \\
\hline $5^{\mathrm{b}}$ & 5.2 & $2.8^{0}$ \\
\hline $5^{\mathrm{b}}$ & 6.5 & $2.8^{0}$ \\
\hline
\end{tabular}

${ }^{\text {a }}$ Performed in panel exerciser (Freestyle Equine Equipment)

${ }^{\mathrm{b}}$ Performed on high speed treadmill (Sato)

After the SET, horses were hand-walked to cool them down for $10 \mathrm{~min}$ to prepare them for the post-exercise muscle biopsy. After biopsy procedures were complete, horses were hosed off and offered ad libitum water while they were monitored for signs of distress.

\section{Sample Collection}

Muscle

Biceps femoris muscle samples were collected the day of the SET, 10 min after the SET (post), and at 24 and $48 \mathrm{~h}$ post SET. Biopsy sites were alternated from each side of the horse (i.e., pre and $24 \mathrm{~h}$ biopsies were performed on the right biceps femoris, while post and $48 \mathrm{~h}$ biopsies were performed on the left biceps femoris). Samples were collected at a consistent depth of approximately 3 to $4 \mathrm{~cm}$. Horses were restrained in 
stocks during the biopsy procedure. A humane lip restraint was utilized in 1 or 2 isolated instances in which the safety of the horse and handlers were in question. One horse was lightly sedated using $1 \mathrm{~mL}$ of $100 \mathrm{mg} / \mathrm{mL}$ xylazine (AnaSed Injection, Lloyd Laboratories, Shenandoah, IL) post jugular venipuncture for horse and handler safety. Immediately prior to the muscle biopsy procedure, an area measuring approximately $7.26 \mathrm{~cm}$ square superior to the biceps femoris was clipped using a \#40 surgical-grade clipper blade. The prepared area was disinfected aseptically using betadine scrub (providine-iodine $5 \%$ ), chlorohexadine ( $2 \%$ chlorohexadine gluconate, Liberty, MO), and $70 \%$ isopropyl alcohol. The site was allowed to sit for $1.5 \mathrm{~min}$ to allow for complete disinfection by the alcohol spray. The area immediately proximal to the muscle sample site was locally anesthetized using between 2.5 and $3 \mathrm{~mL}$ of lidocaine (2\% lidocaine hydrochloride, VetOne, Boise, ID). Lidocaine was administered subcutaneously using a 25G x 1 in needle (Kendall Co., Mansfield, MA) and was allowed to sit for 2 min prior to continuing the procedure.

The muscle biopsy procedure used was adapted from the protocol described by Tarnopolsky et al. (2011) and Snow and Guy (1976). A 5 mm Bergstrom (Stille, Stockholm, Sweden) biopsy needle was fit with $76 \mathrm{~cm} \mathrm{I.V.} \mathrm{tubing} \mathrm{(Abbott} \mathrm{Laboratories,}$ North Chicago, IL), a $14.9 \mathrm{~cm} 5 \mathrm{~mL}$ plastic transfer pipette with the bulb removed (VWR International, West Chester, PA), and attached to a $60 \mathrm{~mL}$ syringe (Coviden, Minneapolis, MN). A small drop of sterile lubricant (Priority Care, Elgin, IL) was added to the internal sleeve of the biopsy needle. This was done immediately prior to the procedure to create a tight seal between the internal and external biopsy needle sleeve. In 
between biopsy procedures, the biopsy needle was stored in a half-water, halfchlorohexidine solution in a disposable medical tray with lid to provide as sterile an environment as possible. Excess solution was removed prior to the biopsy procedure and application of the sterile lubricant.

A $5 \mathrm{~mm}$ stab incision was made using a \#10 scalpel through the center of the previously anesthetized skin and fascia. The biopsy needle was inserted approximately 3 to $4 \mathrm{~cm}$ deep through the incision. The inner cutting sleeve was retracted $3 \mathrm{~cm}$ from the outer sleeve to expose the inner hollow needle surface. Suction was applied when the inner sleeve was retracted to draw muscle tissue into the biopsy needle. The inner sleeve cut the muscle sample by closing the inner sleeve over the hollow needle surface with a twisting motion. Three muscle cut samples were taken at a $0^{0}$ position, $90^{\circ}$ to the left, and $90^{\circ}$ to the right as outlined in Figure 1. 
Figure 1. Biopsy Sample Cuts

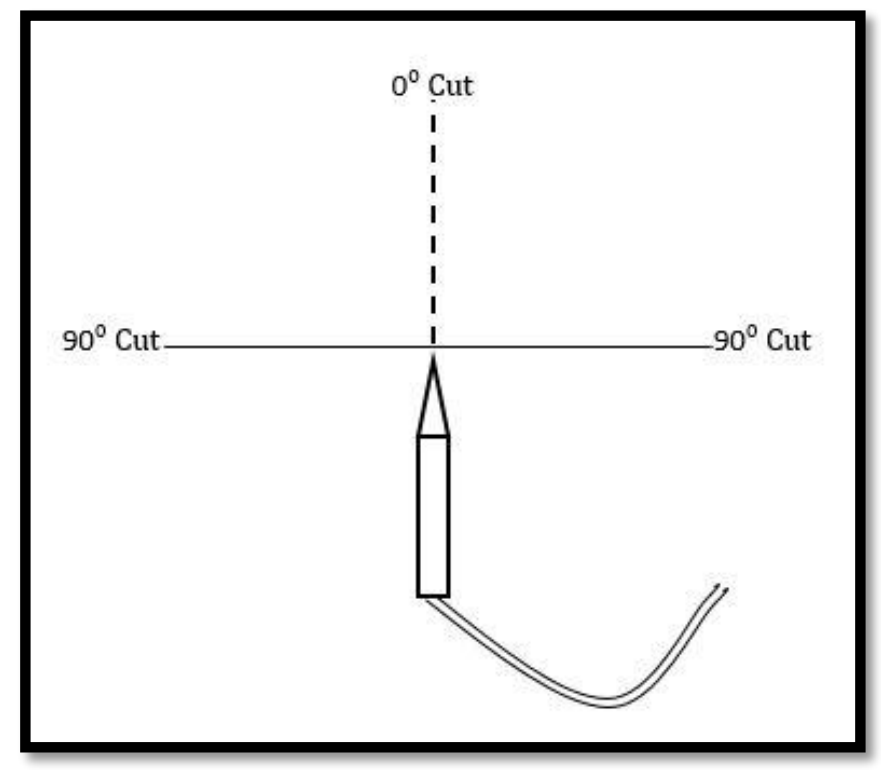

Concluding the sampling procedure, the entire apparatus was removed from the horse and an ice compress and pressure were applied to the area. When the incision was no longer bleeding, adhesive suture material (FutureGlue, Ranch Cucamonga, CA) was applied to keep the incision closed. This was done by pinching the incision site closed and applying a thin layer of surgical adhesive to the touching tissue edges. No sutures were necessary to close any of the incisions. To prevent infection, a topical antimicrobial cream (Silver Sulfadiazine $1 \%$, Ascend Laboratories LLC, Montvale, NJ) was applied after the surgical adhesive dried. Horses were monitored for lameness, infection, and swelling over the next several days. In the instance of swelling, a topical ointment (CutHeal Multi-Care Wound Care, Cut Heal Animal Care Products, Inc., Cedar Hill, TX) 
was applied to keep prevent the area from drying out and to promote healing. There were no long term instances of swelling.

After each biopsy procedure, the muscle sample was placed into a $2 \mathrm{~mL}$ cryogenic vial (VWR, West Chester, PA) and was immediately submerged in liquid nitrogen. The samples were kept submerged in liquid nitrogen until they were stored at $80^{\circ} \mathrm{C}$ until analysis.

Blood

Blood samples were collected by repeated jugular venipuncture into EDTA sterile vacutainer tubes (Becton, Dixon and Company, Franklin Lakes, NJ) at the time of each biopsy. Blood samples were immediately centrifuged at $2500 \mathrm{rpm}$ for $12 \mathrm{~min}$. Serum was then pipetted into labeled $1.5 \mathrm{~mL}$ micro-centrifuge tubes (VWR) and were placed on ice in an insulated cooler until they were transported to storage at $-20^{\circ} \mathrm{C}$ until analysis.

\section{Sample Analysis}

Feed Analysis

Grab samples of all 3 diets were taken either directly from each $22.7 \mathrm{~kg}$ feed bag or mixed in their proper proportions (i.e., HS diet mix of $56 \%$ Strategy GX and $44 \%$ SFC) before a sample was taken. Grab samples were taken with each new batch of feed. The samples were compiled, mixed, and frozen until a sub sample was shipped for analysis. A representative hay sample was taken for each of the 2 hay batches utilized during the study using a hay bale sampler. The 3 experimental diet samples and 2 hay samples were analyzed for \% moisture, DM, CP, ADF, NDC, starch, crude fat via acid 
hydrolysis (grain sample only), TDN, NFC (hay sample only), Ca, P, Mg, K, and DE Mcal/kg by Dairy One Inc. (Ithaca, NY).

Glycogen

Muscle samples were analyzed at the Texas A\&M Department of Health and Kinesiology Muscle Biology Laboratory in College Station, TX. Glycogen concentration of muscle samples were analyzed utilizing a commercial glycogen colorimetric/fluorometric ELISA kit (Biovision Inc., Milipitas, CA). Frozen muscle samples were weighed and an average sub-sample of $15.52 \mathrm{mg}$ of wet tissue was removed for analysis. Individual sub-sample weights were recorded to account for variations in glycogen amount per sample size during statistical analysis. The thawed sub-samples were diluted with $200 \mu \mathrm{L}$ of distilled water and homogenized. The samples were then boiled covered for $10 \mathrm{~min}$ to inactivate enzymes and centrifuged at $18,000 \mathrm{G}$ at $4{ }^{\circ} \mathrm{C}$ for $10 \mathrm{~min}$. Five $\mu \mathrm{L}$ of the supernatant was pipetted, in duplicate, onto a 96 -well plate. The hydrolysis buffer was added so the total volume of each well was $50 \mu \mathrm{L}$. The hydrolysis enzyme mix was added to each well at a volume of $2 \mu \mathrm{L}$ per well. The well was then incubated for $30 \mathrm{~min}$ at room temperature prior to adding $50 \mu \mathrm{L}$ of reaction mix, containing development buffer, development enzyme, and OxiRed probe, to each sample well. The well was incubated in the dark at room temperature for $30 \mathrm{~min}$ before the absorbance was measured on a well reader (Beckerman Coulter, Pasadena, CA) at $595 \mathrm{~nm}$. After the initial incubation reading, the well was again incubated in the dark for $2 \mathrm{~h}$ for a $2 \mathrm{~h}$ incubation reading. After plate precision was determined with interassay and intrassay covariance $(\mathrm{CV})$, it was decided that the $2 \mathrm{~h}$ plate readings were more 
precise and should be utilized for further discussion of glycogen results. The well reader was calibrated within $1 \mathrm{yr}$ of the assay to be accurate up to 1 absorbance unit.

The readings were internally validated for precision using a glycogen standard value. This was prepared by diluting the glycogen standard to $0.2 \mathrm{mg} / \mathrm{mL}$ by adding 10 $\mu \mathrm{L}$ of the standard to $90 \mu \mathrm{L}$ of distilled water and mixing well. The diluted glycogen standard was added to a series of wells in duplicate with $0,2,4,6,8$, and $10 \mu \mathrm{L}$. The volume of each standard well was adjusted to $50 \mu \mathrm{L} /$ well with hydrolysis buffer to generate $0.0,0.4,0.8,1.2,1.6$, and $2.0 \mu \mathrm{g}$ per well of the glycogen standard. The remainder of the preparation process was identical to sample preparation detailed previously.

The kit measured a product produced by the glucoamylase hydrolysis of glycogen that reacted with the OxiRed Probe. The assay detected glycogen from 0.0004 to $2 \mathrm{mg} / \mathrm{mL}$. The 5 glycogen standard wells were used to create a standard curve. The glycogen concentration (wet weight in $\mu \mathrm{g}$ ) of each muscle sample was calculated from the regression of the standard curve.

Three 96-well plates were utilized to run the samples. Three horses were assigned per plate with all 4 muscle samples from each diet run in duplicate on the same plate. This took out the variable of differences between plates affecting an individual horse's results. Additionally, the plate design left room for 1 extra horse diet to be run in duplicate on every plate. This allowed for an internal control across plates. 
Blood

Serum samples collected during the study were analyzed by the Texas A\&M Veterinary Medical Diagnostic Laboratory in College Station, TX. Samples were analyzed for total serum protein (TSP), albumin (AB), P, glucose (GLU), blood urea nitrogen $(\mathrm{BUN})$, creatinine $(\mathrm{SCr})$, total bilirubin $\left(\mathrm{B}_{\mathrm{T}}\right)$, bilirubin direct $\left(\mathrm{B}_{\mathrm{D}}\right)$, creatine kinase (CK), aspartate aminotransferase (AST) or serum glutamic-oxaloacetic transaminase (SGOT), globulin (GLB), albumin/globulin (A/G) ratio, and gammaglutamyl transpeptidase (GGT).

Lactate was analyzed at the Texas A\&M Department of Health and Kinesiology Muscle Biology Laboratory in College Station, Texas utilizing the YSI 2900 Series Biochemistry Analyzer (YSI Inc./Xylem Inc., Yellow Springs, OH). New system buffer (YSI 2357), calibrator dextrose/lactate standard of $2.5 \mathrm{~g} / \mathrm{L}$ dextrose and $0.5 \mathrm{~g} / \mathrm{L}$ lactate (YSI 2776), linearity standard (YSI 1530), and L-lactate membranes (YSI 2329) were purchased from YSI Inc./Xylem Inc. (Yellow Springs, OH) and were system validated prior to sample analysis. Serum samples were thawed on ice and were individually sampled. The sipper self-calibrated every 5 samples and each sample was taken in duplicate to safeguard against biochemical analyzer or sampling error.

\section{Statistical Analysis}

Data was analyzed using the PROC MIXED program in SAS version 9.4 (2015). The model included fixed effects of period, treatment, and time $\mathrm{x}$ treatment interaction; horse was included as a random block effect. Horse within treatment served as the subject of repeated measures, and the covariance structure was specified using the spatial 
power function to account for unequal time spacing of measurements. Differences will be considered significant when $\mathrm{P} \leq 0.05$. 


\section{CHAPTER IV}

\section{RESULTS}

\section{Dietary Starch Intake}

Daily dietary starch intake was first determined by the equation DE Mcal/d = $(0.0333 \times \mathrm{kg} \mathrm{BW}) \times 1.2(\mathrm{NRC}, 2007)$. The average weight of the horses was obtained (463 kg), and horses did not significantly change weight throughout the project (Table 7); thus, intakes did not differ from horse to horse. Hay was fed at $1 \% \mathrm{BW}$ (as fed) and the remaining calories (average of $10.03 \mathrm{Mcal}$ ) not met through hay were fed in concentrate, which averaged $3.293 \mathrm{~kg} / \mathrm{d}$. The amount of concentrate was then multiplied by the concentration of starch to obtain daily dietary starch intake. The results of the analysis (Table 8) show that the HS diet had a greater starch concentration than MS and LS, and that MS had a greater starch concentration than LS.

Table 7. Average Body Weight Throughout Project

\begin{tabular}{|l|l|}
\hline Horse ID & Average BW (kg) \\
\hline Y106 & 494.21 \\
\hline Y11 & 464.32 \\
\hline Y128 & 498.05 \\
\hline B720 & 447.50 \\
\hline B730 & 441.14 \\
\hline B726 & 481.70 \\
\hline W420 & 409.43 \\
\hline W407 & 461.14 \\
\hline W6 & 468.86 \\
\hline Average & 462.92 \\
\hline
\end{tabular}


Table 8. Average Dietary Starch Intake/d, Including Treatment and Hay (As Fed)

\begin{tabular}{l|l}
\hline Diet & Starch Intake (g/d) \\
\hline High Starch (HS) & $1,206.67$ \\
\hline Medium Starch (MS) & 844.61 \\
\hline Low Starch (LS) & 263.13 \\
\hline
\end{tabular}

\section{Diet Transition Order}

Horses were randomly placed into 3 groups with $n=3$ per group and were assigned 1 of the 3 experimental diets. Horses were always transitioned from HS to LS to MS (Table 4) throughout the course of the project to prevent any adverse reactions to starch amount. There were 3 dietary sequences throughout the project. The diet transition orders were: Sequence A (HS-LS-MS), Sequence B (LS-MS-HS), and Sequence C (MS-HS-LS). There was no effect of sequence $(\mathrm{P}=0.80)$ or sequence by period $(\mathrm{P}=0.80)$ on glycogen.

\section{Heart Rate}

Heart rate (HR) was recorded before the 30 min trot on a panel exerciser, after working on a panel exerciser, immediately before the start of the treadmill portion (time 0), 2 min into a treadmill exercise, every 5 min until the completion of the SET, and 10 min after recovery, where horses were hand walked to cool down (Table 9, Figure 2). The maximum $\mathrm{HR}\left(\mathrm{HR}_{\max }\right)$ was also recorded, but was not significant $(\mathrm{P}=0.08)$. Least squares means (LSM) indicated a negative correlation with glycogen and a positive 
correlation with maximum heart rate $\left(\mathrm{HR}_{\max }\right)$. There were significant differences within diets at different time points, but not between diets at the same time points.

Table 9. Mean Heart Rate $( \pm$ SE*)

\begin{tabular}{|c|c|c|c|c|c|c|}
\hline \multicolumn{3}{|c|}{ High Starch ${ }^{12}$} & \multicolumn{2}{|c|}{ Medium Starch $^{1}$} & \multicolumn{2}{|c|}{ Low Starch ${ }^{2}$} \\
\hline $\begin{array}{l}\text { Sample } \\
\text { Time }\end{array}$ & $\begin{array}{l}\text { Beats Per Min } \\
\quad(\mathrm{bpm})\end{array}$ & $\mathrm{SE}^{*}$ & $\begin{array}{l}\text { Beats Per Min } \\
\quad(\mathrm{bpm})\end{array}$ & $\mathrm{SE}^{*}$ & $\begin{array}{l}\text { Beats Per Min } \\
\quad(\mathrm{bpm})\end{array}$ & $\mathrm{SE}^{*}$ \\
\hline Pre† & $43.11^{\mathrm{a}}$ & 3.13 & $43.56^{\mathrm{a}}$ & 2.85 & $48.00^{\mathrm{a}}$ & 2.59 \\
\hline Post† & $78.00^{\mathrm{b}}$ & 5.61 & $66.78^{b}$ & 2.01 & $78.11^{\mathrm{b}}$ & 4.58 \\
\hline 0 & $62.11^{\mathrm{c}}$ & 7.38 & $65.78^{b}$ & 7.27 & $69.78^{b}$ & 6.35 \\
\hline 2 & $87.00^{\mathrm{b}}$ & 3.11 & $83.22^{\mathrm{b}}$ & 1.69 & $85.33^{\mathrm{c}}$ & 4.27 \\
\hline 7 & $103.67^{d}$ & 3.47 & $97.89^{c}$ & 4.67 & $104.67^{d}$ & 6.78 \\
\hline 13 & $117.00^{\mathrm{d}}$ & 3.47 & $104.22^{c}$ & 3.10 & $114.00^{\mathrm{d}}$ & 4.42 \\
\hline 18 & $148.44^{\mathrm{e}}$ & 4.88 & $151.33^{\mathrm{d}}$ & 6.93 & $144.56^{\mathrm{e}}$ & 3.48 \\
\hline 23 & $147.22^{\mathrm{e}}$ & 7.12 & $147.89^{d}$ & 2.72 & $150.33^{\mathrm{e}}$ & 2.03 \\
\hline 27 & $161.57^{\mathrm{e}}$ & 7.88 & $160.00^{d}$ & 6.16 & $163.63^{\mathrm{e}}$ & 3.65 \\
\hline Recovery & $72.56^{\mathrm{c}}$ & 1.61 & $71.00^{\mathrm{b}}$ & 4.07 & $68.33^{\mathrm{b}}$ & 3.14 \\
\hline
\end{tabular}

*SE $=$ Standard error within diet

${ }^{1,2}$ Diets not sharing the same superscript differ $(\mathrm{P}<0.05)(\mathrm{SE} \pm 2.81)$ a,b,c,d,e,f Values in the same column not sharing the same superscript differ $(\mathrm{P}<0.05)$ 
Figure 2. Mean Heart Rate $\left( \pm \mathrm{SE}^{*}\right)$

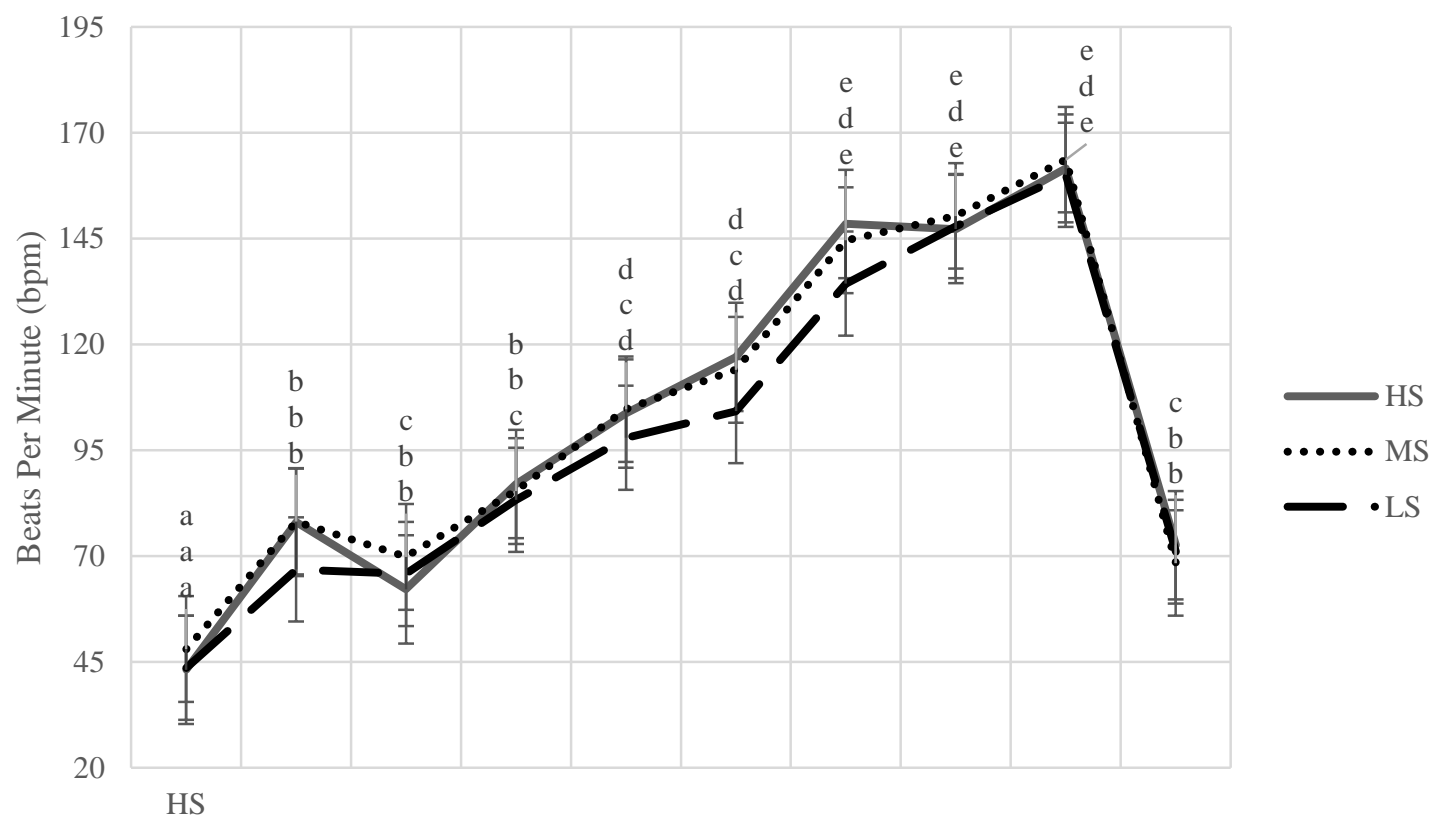

Minutes

$* \mathrm{SE}=$ Standard error

a,b,c,d,e Values in the same diet not sharing the same superscript differ $(\mathrm{P}<0.05)$

Data labels presented top to bottom with HS, MS, and LS

$\uparrow$ Performed in panel exerciser (Freestyle Equine Equipment)

§Hand walked 10 min to cool down

\section{SET Stop Time}

On 4 occasions horses fatigued early during the treadmill portion of the SET and could not keep pace with the treadmill, meaning they could no longer safely complete the duration of the 27 min treadmill portion of the SET. Horse Y106 quit early during each SET regardless of diet, quitting at 23:30, 20:20, and 20:00 min for HS, MS, and LS, respectively. Horse W420 quit at 25:25 min for HS. There was no significance $(\mathrm{P}=$ 0.99) between quit time and treatment for HS, MS, and LS. Additionally, there was no 
significance between quit time and $\mathrm{HR}_{\max }(\mathrm{P}=0.89)$ or quit time on post exercise rectal temperature $(\mathrm{P}=0.29)$.

\section{Skeletal Muscle Glycogen Concentration}

The average skeletal muscle glycogen concentration was incubated for $2 \mathrm{~h}$ prior to the initial reading. The glycogen concentration with the $2 \mathrm{~h}$ incubation period taken at rest, time $0,24 \mathrm{~h}$, and $48 \mathrm{~h}$ post SET are presented in Table 10 and Figure 3 . There was a significant effect on period $(P=0.002)$ and time $(P=0.02)$, with no significant effect on treatment $(\mathrm{P}=0.13)$. The difference of least squared means showed a significant $(\mathrm{P}=$ 0.04) difference between LS and MS in terms of average glycogen concentration. When period effect was taken into consideration, the differences of least squares means (DLSM) was utilized to further analyze treatment $\mathrm{x}$ time interactions. There was a significant difference between HS and MS at rest $(\mathrm{P}=0.009)$ and $\mathrm{HS}$ and LS at $24 \mathrm{~h}$ post SET $(\mathrm{P}=0.04)$. Overall, the $2 \mathrm{~h}$ incubation averaged lower levels of glycogen numerically but with a lower standard error than the initial incubation. The results of the initial incubation can be found in the appendix. 
Table 10. Mean Skeletal Muscle Glycogen Concentration ( $\mu \mathrm{g} / \mathrm{mg}$ wet weight) $2 \mathrm{~h}$ Incubation $\left( \pm \mathrm{SE}^{*}\right)$

\begin{tabular}{l|c|c|c|c|c|c}
\hline \multicolumn{3}{|c}{ High Starch $^{12}$} & \multicolumn{3}{c}{ Medium Starch $^{2}$} & \multicolumn{2}{c}{ Low Starch $^{1}$} \\
\hline $\begin{array}{l}\text { Sample } \\
\text { Time }\end{array}$ & Concentration & SE* & Concentration & SE* & Concentration & SE* $^{*}$ \\
\hline Rest & $10.25^{\mathrm{a} \dagger}$ & 0.93 & $8.28^{\mathrm{a} \dagger}$ & 0.97 & $9.11^{\mathrm{a}}$ & 0.78 \\
\hline $\begin{array}{l}\text { Post } \\
\text { Exercise }\end{array}$ & $7.38^{\mathrm{b}}$ & 0.48 & $7.77^{\mathrm{a}}$ & 0.29 & $8.55^{\mathrm{a}}$ & 0.64 \\
\hline $24 \mathrm{~h}$ & $7.68^{\mathrm{b} \dagger}$ & 0.79 & $8.26^{\mathrm{a}}$ & 0.58 & $9.52^{\mathrm{a} \dagger}$ & 0.68 \\
\hline $48 \mathrm{~h}$ & $8.84^{\mathrm{ab}}$ & 0.50 & $9.16^{\mathrm{a}}$ & 0.53 & $9.29^{\mathrm{a}}$ & 0.55 \\
\hline
\end{tabular}

$* \mathrm{SE}=$ Standard Error within diet

${ }^{1,2}$ Diets not sharing the same superscript differ $(\mathrm{P}<0.05)(\mathrm{SE} \pm 0.50)$

${ }^{a, b}$ Values in the same column not sharing the same superscript differ $(\mathrm{P}<0.05)$

$\dagger$ Values in the same row differ $(\mathrm{P}<0.05)$

Figure 3. Mean Skeletal Muscle Glycogen Concentration $2 \mathrm{~h}$ Incubation $\left( \pm \mathrm{SE}^{*}\right)$

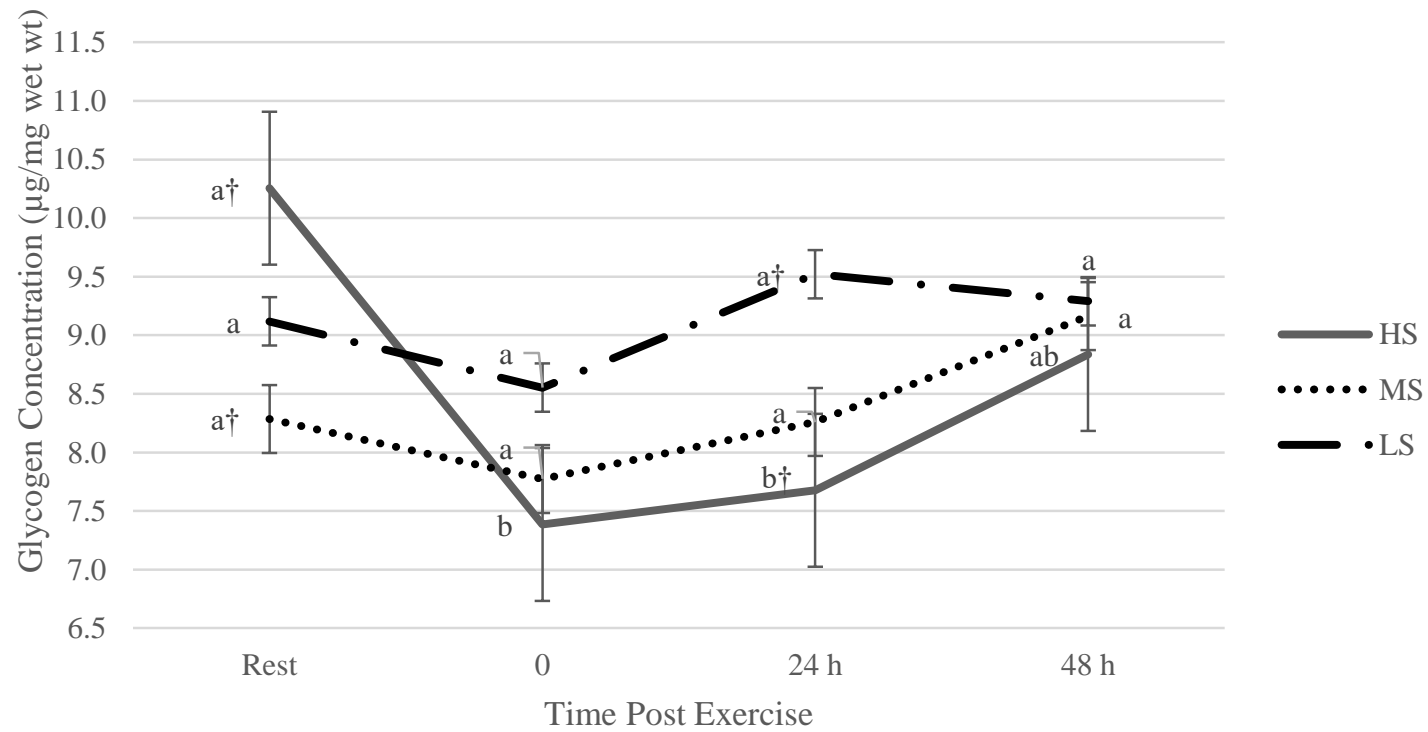

*SE $=$ Standard error

${ }^{a, b}$ Values in the same diet not sharing the same superscript $\operatorname{differ}(\mathrm{P}<0.05)$

$\dagger$ Values at the same time point differ $(\mathrm{P}<0.05)$ 
Skeletal glycogen depletion and replenishment percentages for the $2 \mathrm{~h}$ incubation were calculated in the same manner as the percentages for the initial incubation period (Table 11, Figure 4). High starch had the greatest depletion of glycogen post exercise with $27.98 \%$. High starch repleted $2.83 \%$ from post exercise to $24 \mathrm{~h}$ post (difference between percentage changes of post and $24 \mathrm{~h}$ ). High starch repleted $11.31 \%$ between 24 and $48 \mathrm{~h}$. Medium starch had similar depletion of glycogen to LS post exercise with 6.18 $\%$ and $6.19 \%$, respectively. Medium starch replenished $5.86 \%$ from post exercise to 24 h. Medium starch further replenished $10.91 \%$ and had $10.59 \%$ greater glycogen concentration $48 \mathrm{~h}$ post exercise than at rest. Low starch replenished $10.62 \%$ from post exercise to $24 \mathrm{~h}$, resulting in an increase in glycogen concentration $4.43 \%$ over resting glycogen levels at $24 \mathrm{~h}$. Between 24 and $48 \mathrm{~h}$, LS depleted further by $6.31 \%$, resulting in a glycogen concentration $1.88 \%$ higher than the resting glycogen concentration.

Horses on the HS diet utilized the greatest amount of glycogen. The MS and LS diet experienced similar patterns of depletion post exercise. The MS diet resulted in the greatest concentration of glycogen $48 \mathrm{~h}$ post exercise. The percentage of skeletal muscle glycogen at $48 \mathrm{~h}$ compared to resting concentration was MS > LS > HS. 
Table 11. Mean Skeletal Muscle Glycogen Percentage Depletion/Repletion $2 \mathrm{~h}$ Incubation $\left( \pm \mathrm{SE}^{*}\right)$

\begin{tabular}{l|c|c|c|c|c|c}
\hline \multicolumn{3}{c}{ High Starch $^{12}$} & \multicolumn{3}{c}{ Medium Starch $^{2}$} & \multicolumn{2}{c}{ Low Starch $^{1}$} \\
\hline $\begin{array}{l}\text { Sample } \\
\text { Time }\end{array}$ & $\begin{array}{c}\% \text { of Rest } \\
\text { Value }\end{array}$ & $\begin{array}{c}\% \\
\text { Change }\end{array}$ & $\begin{array}{c}\text { of Rest } \\
\text { Value }\end{array}$ & $\begin{array}{c}\% \\
\text { Change }\end{array}$ & $\begin{array}{c}\text { \% of Rest } \\
\text { Value }\end{array}$ & $\%$ Change \\
\hline Rest & $100^{\mathrm{a} \dagger}$ & 0 & $100^{\mathrm{a} \dagger}$ & 0 & $100^{\mathrm{a}}$ & 0 \\
\hline $\begin{array}{l}\text { Post } \\
\text { Exercise }\end{array}$ & $72.02^{\mathrm{b}}$ & 27.98 & $93.82^{\mathrm{a}}$ & 6.18 & $93.81^{\mathrm{a}}$ & 6.19 \\
\hline $24 \mathrm{~h}$ & $74.85^{\mathrm{b} \dagger}$ & 25.15 & $99.68^{\mathrm{a}}$ & 0.32 & $104.43^{\mathrm{a} \dagger}$ & -4.43 \\
\hline $48 \mathrm{~h}$ & $86.16^{\mathrm{ab}}$ & 13.84 & $110.59^{\mathrm{a}}$ & -10.59 & $101.88^{\mathrm{a}}$ & -1.88 \\
\hline
\end{tabular}

*SE $=$ Standard Error within diet

${ }^{1,2}$ Diets not sharing the same superscript differ $(\mathrm{P}<0.05)(\mathrm{SE} \pm 0.50)$

${ }^{\mathrm{a}, \mathrm{b}}$ Values in the same column not sharing the same superscript differ $(\mathrm{P}<0.05)$

$\dagger$ Values in the same row differ $(\mathrm{P}<0.05)$

Figure 4. Glycogen Percentage Depletion/Repletion

\section{$2 \mathrm{~h}$ Incubation $\left( \pm \mathrm{SE}^{*}\right)$}

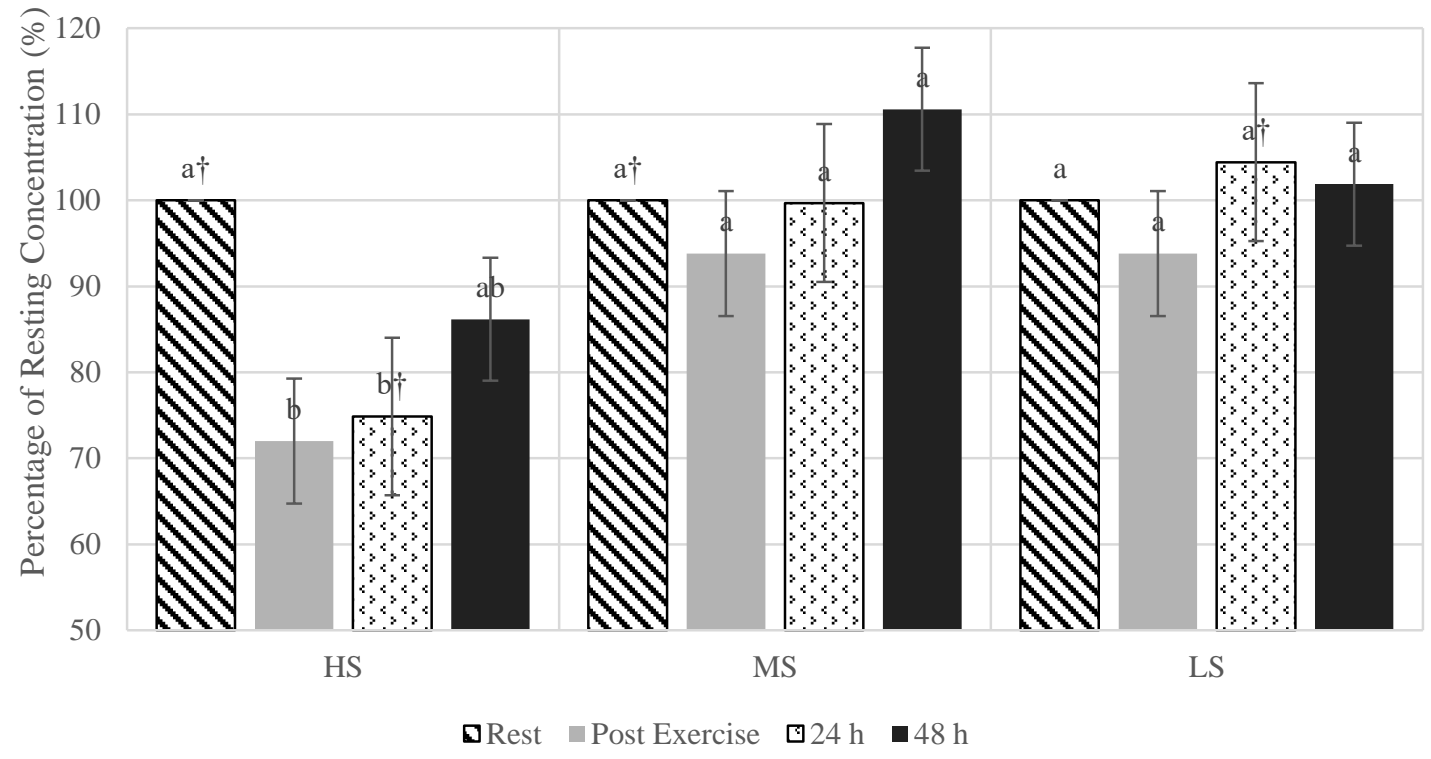

*SE= Standard error

${ }^{a, b}$ Values in the same diet not sharing the same superscript differ $(\mathrm{P}<0.05)$

$\dagger$ Values at the same time point differ $(\mathrm{P}<0.05)$ 


\section{Glycogen Assay Kit Plate Internal Controls}

Three plates were used to run glycogen samples, with 3 horses and each of their dietary treatments run in duplicate on the 96-well plate. This was done to mitigate plate to plate differences. This left room for 1 horse and dietary treatment to be used as an internal control. Horse B720 and the HS dietary treatment was run in duplicate on each of the 3 plates, with precision measured with both an interassay CV and intrassay CV. Interassay $\mathrm{CV}$ returned $20 \%$ and $18 \%$ for initial incubation period and $2 \mathrm{~h}$ incubation period, respectively. Intrassay CV returned $22 \%, 24 \%$, and $22 \%$ for the initial incubation plates, and $21 \%, 17 \%$, and $17 \%$ for the $2 \mathrm{~h}$ incubation plates.

\section{Blood Markers}

\section{Lactate Concentration}

There was a significant effect of time $(\mathrm{P}<0.0001)$ on lactate, with a close statistical similarity between treatments $(\mathrm{P}=0.98)$. Average mean lactate values from LSM were 2.93, 2.87, and 2.84 for HS, MS, and LS, respectively. When graphed, blood lactate concentration is considerably similar across diets, indicating a nearly identical energy expenditure during the SET regardless of diet (Table 12, Figure 5).

Due to the similarities between the blood lactate concentrations across diets $(\mathrm{P}=$ 0.98), a prediction equation was formed to estimate the expected level of lactate from immediately after exercise to $24 \mathrm{~h}$ post exercise. The mean at each time point for each diet was pooled together to form the prediction equation. The prediction equation yields blood lactate concentration $(\mathrm{mmol} / \mathrm{L})$ at a given time point from $0 \mathrm{~min}$ to $10 \mathrm{~min}$ and reads: $y=0.002 x^{2}-0.3102 x+6.6874$. 
Table 12. Mean Blood Lactate Concentration (mmol/L) ( $\left.\pm \mathrm{SE}^{*}\right)$

\begin{tabular}{l|c|c|c|c|c|c}
\hline \multicolumn{3}{|c}{ High Starch } & \multicolumn{2}{c}{ Medium Starch } & \multicolumn{2}{c}{ Low Starch } \\
\hline $\begin{array}{l}\text { Sample } \\
\text { Time }\end{array}$ & Concentration & SE* & Concentration & SE* & Concentration & SE* \\
\hline Rest & $0.52^{\mathrm{a}}$ & 0.08 & $0.60^{\mathrm{a}}$ & 0.05 & $0.59^{\mathrm{a}}$ & 0.07 \\
\hline $0 \mathrm{~min}$ & $6.87^{\mathrm{b}}$ & 1.17 & $6.50^{\mathrm{b}}$ & 0.81 & $6.69^{\mathrm{b}}$ & 0.98 \\
\hline $10 \mathrm{~min}$ & $3.73^{\mathrm{c}}$ & 0.88 & $3.41^{\mathrm{c}}$ & 0.53 & $3.68^{\mathrm{c}}$ & 0.73 \\
\hline $24 \mathrm{~h}$ & $0.61^{\mathrm{a}}$ & 0.04 & $0.67^{\mathrm{a}}$ & 0.05 & $0.66^{\mathrm{a}}$ & 0.06 \\
\hline
\end{tabular}

$* \mathrm{SE}=$ Standard error within diet

${ }^{\mathrm{a}, \mathrm{b}}$ Values in the same column not sharing the same superscript differ $(\mathrm{P}<0.001)$

Figure 5. Mean Lactate Concentration $\left( \pm \mathrm{SE}^{*}\right)$

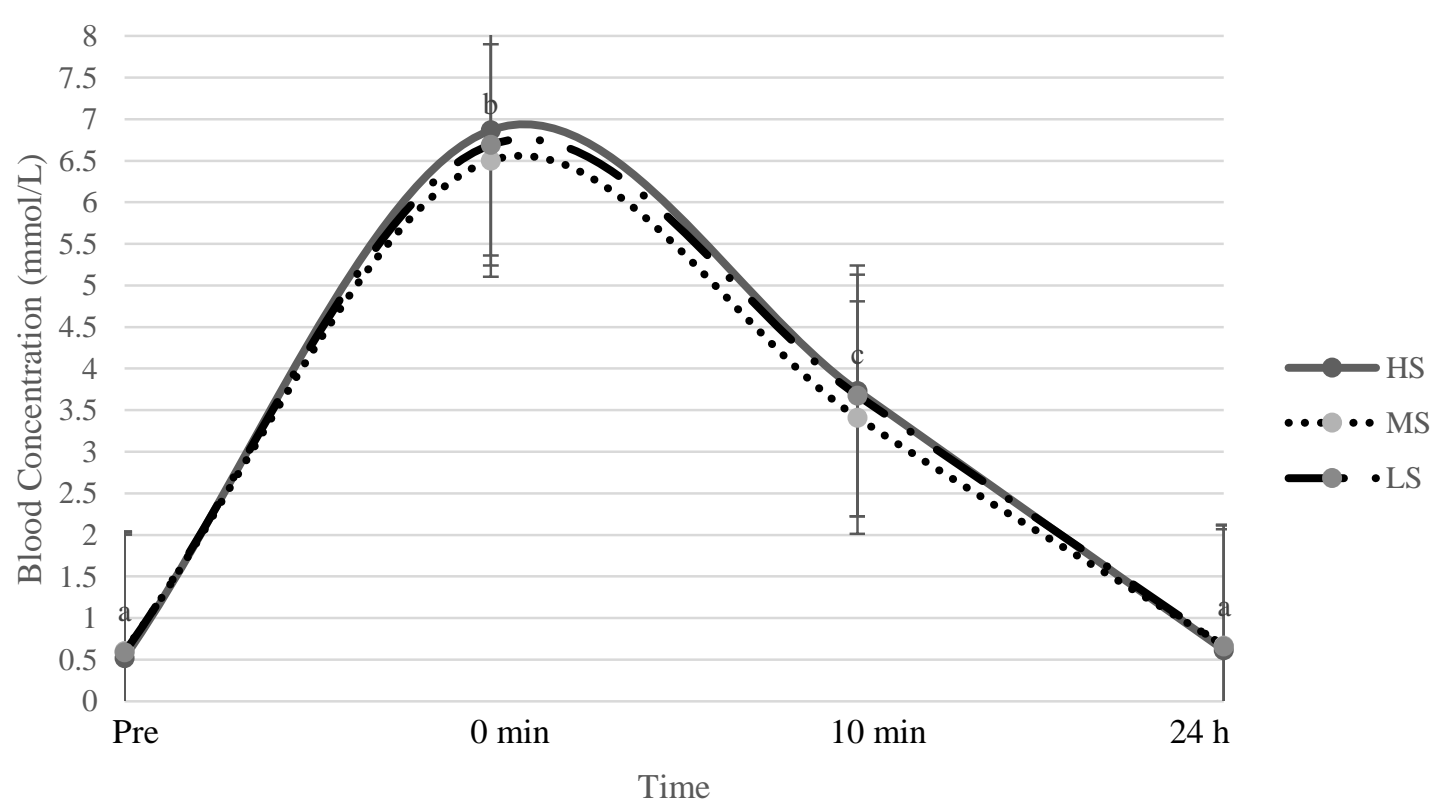

$* \mathrm{SE}=$ Standard error

${ }^{a, b, c}$ Data labels at all time points the same across diets 


\section{Glucose Concentration}

There was a significant effect $(\mathrm{P}<0.0001)$ of time on GLU concentration.

Glucose had a positive correlation with skeletal muscle glycogen concentrations at both the initial and $2 \mathrm{~h}$ incubation. Difference of least square means was not significant $(\mathrm{P}=$ 0.61) between diets, but showed significant $(\mathrm{P}<0.001)$ difference at time points within diets for GLU (Table 13). For the HS diet, there was a significant difference between blood GLU concentrations at rest vs. $0 \min (\mathrm{P}<0.0001), 0$ min vs. $10 \min (\mathrm{P}=0.009), 0$ $\min$ vs. $24 \mathrm{~h}(\mathrm{P}<0.0001)$, rest vs. $10 \min (\mathrm{P}<0.0001)$, and $10 \min$ vs. $24 \mathrm{~h}(\mathrm{P}<$ 0.0001). Blood GLU concentration was not significant $(\mathrm{P}=0.98)$ when comparing rest vs. 24 h. For both MS and LS diets, there was a significant difference between blood GLU concentrations at rest vs. 0 min $(\mathrm{P}<0.0001)$, rest vs. $10 \mathrm{~min}(\mathrm{P}<0.0001), 0 \mathrm{~min}$ vs. $24 \mathrm{~h}(\mathrm{P}<0.0001)$, and $10 \mathrm{~min}$ vs. $24 \mathrm{~h}(\mathrm{P}<0.0001)$. Blood GLU concentration was not significant for MS or LS when comparing rest vs. $24 \mathrm{~h}(\mathrm{P}=0.36 ; \mathrm{P}=067)$ or $0 \mathrm{~min}$ vs. $10 \min (\mathrm{P}=0.16 ; \mathrm{P}=0.86)$, respectively.

Table 13. Mean Blood Glucose Concentration (mg/dL) ( $\left.\pm \mathrm{SE}^{*}\right)$

\begin{tabular}{l|c|c|c|c|c|c}
\hline \multicolumn{3}{|c}{ High Starch } & \multicolumn{3}{c}{ Medium Starch } & \multicolumn{3}{c}{ Low Starch } \\
\hline $\begin{array}{l}\text { Sample } \\
\text { Time }\end{array}$ & Concentration & SE* & Concentration & SE* & Concentration & SE* \\
\hline Rest & $86.67^{\mathrm{a}}$ & 3.21 & $83.78^{\mathrm{a}}$ & 3.24 & $89.22^{\mathrm{a}}$ & 3.52 \\
\hline $0 \mathrm{~min}$ & $111.11^{\mathrm{b}}$ & 2.74 & $109^{\mathrm{b}}$ & 4.06 & $112.33^{\mathrm{b}}$ & 4.42 \\
\hline $10 \mathrm{~min}$ & $116.33^{\mathrm{c}}$ & 3.85 & $111.78^{\mathrm{b}}$ & 3.03 & $112.78^{\mathrm{b}}$ & 4.96 \\
\hline $24 \mathrm{~h}$ & $86.78^{\mathrm{a}}$ & 1.46 & $87.56^{\mathrm{a}}$ & 1.56 & $87.89^{\mathrm{a}}$ & 1.59 \\
\hline
\end{tabular}

$* \mathrm{SE}=$ Standard error within diet

${ }^{a, b}$ Values in the same column not sharing the same superscript differ $(\mathrm{P}<0.001)$ 


\section{Blood Urea Nitrogen Concentration}

There was a significant effect of time $(\mathrm{P}<0.0006)$ on BUN. Differences of least squares means indicated that $\mathrm{BUN}$ had the greatest significant difference $(\mathrm{P}=0.03)$ between LS and HS (Table 14, Figure 6). Low starch and HS were significantly different at all time points except at $0 \min (\mathrm{P}=0.06)$.

Table 14. Mean Blood Urea Nitrogen Concentration (mg/dL) ( $\left.\pm \mathrm{SE}^{*}\right)$

\begin{tabular}{|c|c|c|c|c|c|c|}
\hline & \multicolumn{2}{|c|}{ High Starch ${ }^{1}$} & \multicolumn{2}{|c|}{ Medium Starch 12} & \multicolumn{2}{|c|}{ Low Starch ${ }^{2}$} \\
\hline $\begin{array}{l}\text { Sample } \\
\text { Time }\end{array}$ & Concentration & $\mathrm{SE}^{*}$ & Concentration & $\mathrm{SE}^{*}$ & Concentration & SE* \\
\hline Rest & $12.78^{\mathrm{a}^{\dagger}}$ & 0.64 & $13.98^{\mathrm{a}}$ & 0.72 & $14.74^{\mathrm{a}^{\dagger}}$ & 0.43 \\
\hline 0 min & $13.50^{\mathrm{a}}$ & 0.56 & $14.65^{\mathrm{ac}}$ & 0.57 & $15.38^{\mathrm{ac}}$ & 0.53 \\
\hline $10 \mathrm{~min}$ & $13.38^{\mathrm{a}^{\dagger}}$ & 0.60 & $15.17^{b}$ & 0.70 & $15.67^{\mathrm{bc} \dagger}$ & 0.51 \\
\hline $24 \mathrm{~h}$ & $13.50^{\mathrm{a}^{\dagger}}$ & 0.94 & $15.22^{b c}$ & 0.67 & $15.70^{\mathrm{bc} \dagger}$ & 0.65 \\
\hline
\end{tabular}

*SE $=$ Standard error within diet

1,2Diets not sharing the same superscript differ $(\mathrm{P}<0.05)(\mathrm{SE} \pm 0.64)$

a,b Values in the same column not sharing the same superscript differ $(\mathrm{P}<0.05)$

$\dagger$ Values in the same row differ $(\mathrm{P}<0.05)$ 


\section{Figure 6. Mean Blood Urea Nitrogen Concentration \\ $\left( \pm \mathrm{SE}^{*}\right)$}

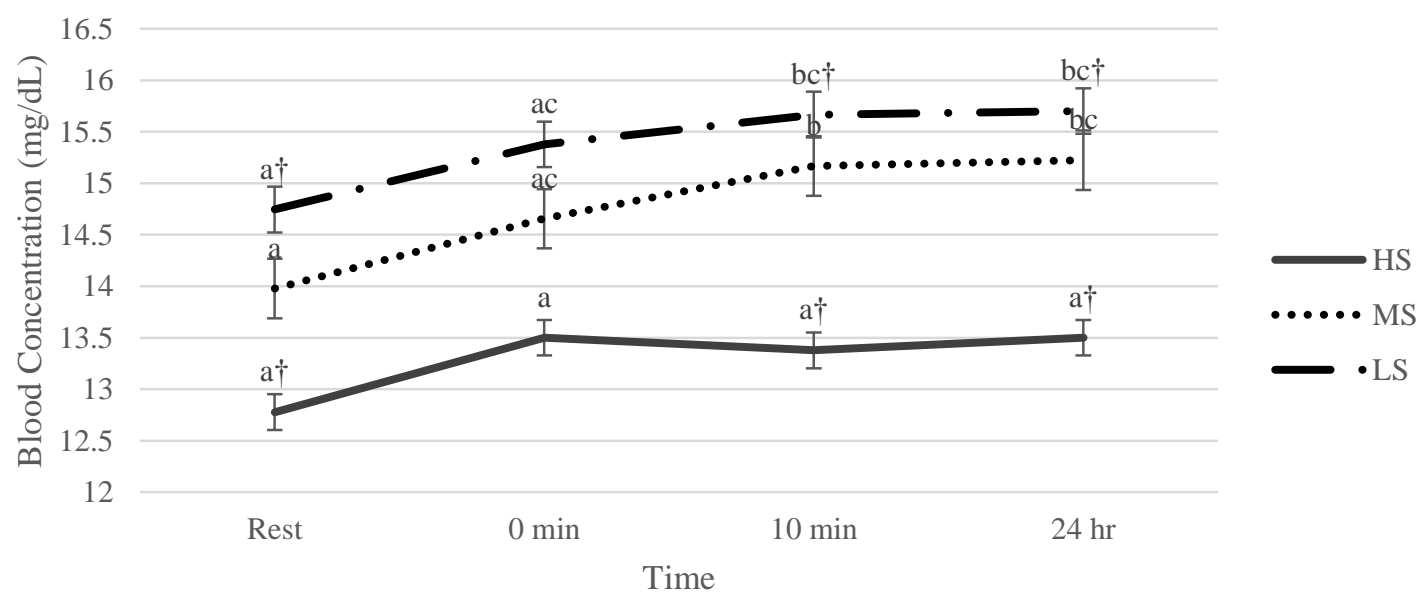

$* \mathrm{SE}=$ Standard error within diet

${ }^{\mathrm{a}, \mathrm{b}}$ Values in the same diet not sharing the same superscript differ $(\mathrm{P}<0.05)$

$\dagger$ Values at the same time point differ $(\mathrm{P}<0.05)$

\section{Additional Blood Markers}

There was a significant effect of time $(\mathrm{P}<0.0001)$ on ALB, AST, SCr, GLB, P, and TSP. Aspartate aminotransferase was the only blood value indicating a negative correlation with glycogen. There was no significant difference $(\mathrm{P}=0.50)$ in terms of dietary treatment for AST. The average values of GLB indicated a positive trend with skeletal muscle glycogen concentration at both the initial and $2 \mathrm{~h}$ incubation. There was a significant effect of time on the $\mathrm{A} / \mathrm{G}(\mathrm{P}=0.0017)$, and $\mathrm{GGT}(\mathrm{P}=0.0020)$ concentration. $\mathrm{B}_{\mathrm{T}}$ and $\mathrm{B}_{\mathrm{D}}$ were the only blood markers that period had a significant effect on, with $(P=0.0019)$ and $(P=0.0073)$, respectively. Creatine kinase was the only blood marker that was not significantly affected by time $(\mathrm{P}=0.12)$. The average values 
of $\mathrm{CK}$ had a positive correlation with skeletal muscle glycogen concentration at both the initial and $2 \mathrm{~h}$ incubation. 


\section{CHAPTER V}

\section{DISCUSSION AND CONCLUSION}

Fatigue in the performance horse industry can lead to loss of performance which subsequently affects loss of award money, breeding demand after retirement, and a potential increase in fatigue-related injuries. Equine athletes performing in events lasting multiple days deplete glycogen stores, which serve as an important fuel for energy during maximal, exhaustive exercise (Harris et al., 1987; Pagan, 1998). Because skeletal muscle glycogen replenishment in the horse is 3 to 4 fold slower than the human, multiday events with multiple bouts of highly anaerobic exercise can take a toll. The slow replenishment of glycogen coupled with a current industry trend of high fat, high fiber, and subsequently low starch, diets might result in premature fatigue due to lower substrate availability and lower resting skeletal muscle glycogen stores (Kline and Albert, 1981; Waller and Lindinger, 2010). The anecdotal evidence of fatigue in horses on low starch diets in the performance horse industry, coupled with recent research evaluating the effects of high vs. low starch diets, led to the primary objective of this project, which was to compare post exercise glycogen replenishment patterns and markers of recovery in horses fed high, medium, and low starch diets (Vonderohe, 2013; Pagan et al., 2015).

\section{Heart Rate}

The average HR throughout the SET was higher on MS than HS and LS, respectively. This is in contrast to other studies where LS diets tended to have consistently higher HR but with no significance (Topliff, 1984; Pagan et al., 1987; 
Vonderohe, 2013). This also is in direct contrast to a study by Spencer et al. (1992), who reported that human subjects with higher resting glycogen concentrations have lower HR vs. subjects with low resting glycogen concentrations. Additional studies have found no trend or difference between HR with fat supplementation (Meyers et al., 1989; Julen, 1994). Numerous studies have reported the effects of lower HR due to omega-3 FA supplementation in both the human and dog (Billman et al., 1997; Peoples et al., 2008; Kang, 2012). The commercial ration utilized for LS contains a fat supplement nugget advertised as containing omega-3 FA. However, analysis of the amount of omega-3 FA present in the diet was not performed.

The increase in HR with increasing exercise intensity across all treatments was expected and agreed with current literature, as the linear relationship between HR and workload effort has been well documented in horses trotting and galloping (Lindholm and Saltin, 1974; Poole and Erickson, 2004; Hodgson et al., 2014). The negative correlation between HR and skeletal muscle glycogen was also expected, as both glycogen utilization and HR increase with increasing exercise intensity (Gollnick et al., 1973b; Nimmo and Snow, 1983; Davie et al., 1999).

\section{SET Stop Time}

On the 4 occasions where horses fatigued early and could not complete the SET, there was no significance $(\mathrm{P}=0.99)$ between quit time and treatment for HS, MS, and LS. The early fatigue seen in W420 was most likely due to obstinacy, as this particular gelding was prone to being difficult to manage at times. Additionally, the SET on which W420 quit was his first SET of the project. Although he had been properly acclimated to 
the treadmill and horses readily adapt to running on a treadmill, W420 had not experienced a run to fatigue (Hodgson, 2008). W420 completed the full SET on the next 2 treatments. Y106 was of more interest, as he fatigued during the SET regardless of diet and was of an agreeable nature. Although not recorded, Y106's respirations seemed more rapid than other horses, which may be a sign of distress despite the absence of exercised induced pulmonary hemorrhage (Hodgson et al., 2014). It was inferred that Y106 had an underlying medical condition that resulted in premature fatigue (Dr. Dennis Sigler, personal communication, November 2014). However, this did not manifest through abnormal $\mathrm{HR}_{\max }$, recovery $\mathrm{HR}$, or blood markers.

\section{Initial Glycogen Incubation vs. 2 h Glycogen Incubation}

Incubation of the well plates for glycogen detection occurred as per the protocol provided in the ELISA kit. The plates were then incubated in the dark for $2 \mathrm{~h}$ prior to the initial reading to allow the glucoamylase in the ELISA kit enough time to hydrolyze large amounts of glycogen for detection with the OxiRed probe (Dr. James Fluckey, personal communication, May 2015). The following discussion of skeletal muscle glycogen concentration refers to results of the $2 \mathrm{~h}$ incubation as these plates proved more accurate with $\mathrm{CV}$ analysis and the SE was lower than in the initial incubation.

Discussion referring to the initial glycogen incubation can be found in the appendix.

\section{Resting Glycogen Concentration}

There was a significant difference $(\mathrm{P}=0.009)$ between the resting skeletal muscle glycogen concentration of HS and MS. This is a different outcome than what was seen in a similar experiment done by Vonderohe (2013), who saw no significant 
differences between diets. The resting concentrations of the present study aligned with the outcome of similar experiments by Topliff (1984) and Pagan et al. (1987), who saw significant differences in the resting glycogen concentration between a control diet and both a high protein and high fat diet. Scott et al. (1992) also found a significant difference in resting glycogen concentration between a control and fat supplemented diet in horses of moderately low, moderate, and moderately high body condition.

Interestingly, the LS diet was not statistically different. This may be explained that the fat in the diet $(13.6 \%)$ was not in a quantity high enough to elicit an effect (Meyers et al., 1989; Oldham et al., 1990). Some differences between which diets are different vs. the work of Topliff (1984) and Pagan et al. (1987) could be the fact that horses were conditioned while on different diets, so the results could be a combination of treatment and exercise effect.

\section{Glycogen Depletion from SET}

The SET used to deplete skeletal muscle glycogen in the present study was designed to deplete both aerobic and anaerobic muscle fibers by having the horses trot for $30 \mathrm{~min}$ on the panel exerciser before being moved to the high speed treadmill for 27 min. The treadmill portion was designed to challenge the preferential fiber recruitment pattern by changing intensities of speed and incline (Valberg, 1986). After exercise, horses on the HS diet depleted the most glycogen (27.98\%), with MS and LS diets experiencing a depletion of $6.18 \%$ and $6.19 \%$, respectively. This depletion in HS indicates HS had greater substrate availability to draw from during highly anaerobic exercise (Kline and Albert, 1981; Pagan et al., 2015). 
Topliff (1984) and Lacombe et al. (1999) reported that depletion of skeletal muscle glycogen by $40 \%$ was needed to induce a decrease in anaerobic capacity in horses though the depletion of muscle fibers. Vonderohe (2013) successfully depleted 50 $\%$ of glycogen in both diets utilizing a SET identical to the present study. However, alternate studies align with the present study's glycogen depletion rate of $27.98 \%$ for the HS diet. A similar SET was utilized by Pagan et al. (1987), McCutcheon et al. (1999) and Waller et al. (2009) with glycogen depletion of $20 \%$ to $30 \%$. Miller and Lawrence (1986) utilized a treadmill protocol of horses carrying $17 \mathrm{~kg}$ at $4.5 \mathrm{~m} / \mathrm{s}$ on a $9 \%$ grade to illicit fatigue, resulting in a $25 \%$ decrease in muscle glycogen concentration. Additionally, Davie et al. (1999) achieved glycogen depletion of $21 \%$ and $29 \%$ using different treadmill protocols to selectively deplete Type I and Type II fibers, respectively.

It is challenging to compare muscle glycogen depletion across studies due to differences between treadmill protocols. Increasing the grade or degree incline on a treadmill is well understood to illicit fatigue quicker than working on the flat. Weighted horses travelling at $4.2 \mathrm{~m} / \mathrm{s}$ on a $9 \%$ grade fatigued at an average of $16.1 \pm 1.6 \mathrm{~min}$, while unweighted horses travelling $3.5 \mathrm{~m} / \mathrm{s}$ to $5.7 \mathrm{~m} / \mathrm{s}$ could complete a $1 \mathrm{~h}$ SET on a treadmill at a $10 \%$ grade (Miller and Lawrence, 1986; Davie et al., 1996). However, markers such as $\mathrm{HR}_{\max }, \mathrm{VO} 2 \mathrm{max}$, and $\mathrm{VLA}_{4}$ (velocity at which blood lactate concentration is $4 \mathrm{mmol} / \mathrm{L}$ ) can be utilized across studies to access fitness (Rainger et al., 1994). Jones and Doust (1996) reported that treadmill test methodology was highly repeatable $(r=0.99)$ which was supported by Seeherman and Morris (1990), who found 
that exercise tests are a reliable source of a horse's metabolic capacity. Hodgson, (2008) also concluded that treadmill exercise tests are reliable in determining why a horse may not be performing at previous levels, and may have uses to determine performance potential as methods become more refined.

The lower skeletal muscle glycogen depletion observed in the MS and LS diets in relation to the HS diet may be explained by the substrate utilized during exercise. The decreased glycogen utilization in the present study aligned with previous findings by Pagan et al. (1987) and Griewe et al. (1989), thus indicating exercise was fueled by a substrate other than glycogen. The mechanism behind this would be fat adaptation, which would result in a preferential utilization of lipids during aerobic exercise, thus sparing glycogen for anaerobic exercise (Meyers et al., 1989; Oldham et al., 1990; Jentjens and Jeukendrup, 2003). When comparing the dietary treatments of the present study, LS had the highest fat content at $13.6 \% \mathrm{DM}$, followed by $9 \%$ and $6.9 \%$ for MS and HS, respectively. The level of fat in both the LS and MS diet would be enough to elicit preferential fat utilization (Hambleton et al., 1981; Meyers et al., 1989). Metabolic adaptations to fat supplementation occur, making lipids a desirable substrate during aerobic metabolism (Lawrence, 1990). Duren et al. (1987) found that horses receiving higher levels of dietary fat had lower levels of circulating triglycerides, suggesting an increase in lipoprotein lipase (LPL) activity. Lipoprotein lipase hydrolyzes TG into 2 FFA and 1 monoacylglycerol molecule. Glycerol is then converted into glucose via gluconeogenesis (Lawrence, 1990; Jose-Cunilleras and Hinchcliff, 2004). Free fatty acids levels were not assessed in the present study. 
Although protein is not a major fuel source during exercise, amino acids (AA) derived from dietary crude protein can be broken down for energy (Lawrence, 1990). Evidence of protein utilization specifically in LS and MS may be indicated by BUN concentrations, as urea is a waste product of protein catabolism. Catabolism of AA begins with deamination and modification of remaining carbon skeletons through gluconeogenesis. Glycogenic AA are degraded to precursors of the citric acid cycle, such as pyruvate. Ketogenic AA are degraded to acetyl-CoA (Horton et al., 2006). When the amine group is removed from an AA, it is converted to ammonia. Ammonia is toxic to cells in large quantities, so ammonia is converted to urea in the liver (Pagan, 1998; Horton et al., 2006). Blood urea travels from the liver to the kidneys, where the urea is excreted in urine.

Throughout the present study, samples of the biceps femoris were taken a total of 12 times, with 6 biopsies per side. Damage to the sampled tissue from previous biopsies (blood vessel damage or scar tissue presence) most likely was not a factor in variations between skeletal muscle glycogen depletion, as Lindholm and Piehl (1974) obtained as many as 20 repeated biopsies from the same horse on the same day without any adverse results.

\section{Glycogen Replenishment}

There was a significant effect of period $(\mathrm{P}=0.002)$ and time $(\mathrm{P}=0.02)$, with no significant effect $(\mathrm{P}=0.13)$ of treatment. However, when DLSM was utilized to analyze treatment $\mathrm{x}$ time interactions using period effect, there was a significant difference $(\mathrm{P}=$ 0.009) between HS and LS at $24 \mathrm{~h}$ post exercise. The lack of statistical significance at 
other time points during replenishment can be attributed to sample to sample variations, as was seen by Vonderohe (2013).

Glycogen replenishment in the HS diet was incremental in fashion but was significantly lower than the LS diet $24 \mathrm{~h}$ post exercise with glycogen replenished to $74.85 \%$ of resting values and $104.43 \%$, respectively. Glycogen repletion for HS at $24 \mathrm{~h}$ was similar to values reported by Vonderohe (2013) at the $6 \mathrm{~h}$ mark, indicating that glycogen repletion was slower in the present study. However, HS diet glycogen repletion was greater at $24 \mathrm{~h}$ than the repletion reported by Hyyppä et al. (1997) at $24 \mathrm{~h}$ and $48 \mathrm{~h}$. This was in contrast to findings by Jose-Cunilleras et al. (2006) who found that horses fed high $\mathrm{CHO}$ had higher skeletal muscle glycogen concentrations at $24 \mathrm{~h}$ than their pre exercise values. Although this is untrue for the HS diet in the present study, the MS diet replenished to $99.68 \%$ of the resting skeletal muscle glycogen concentration at $24 \mathrm{~h}$.

Glycogen replenishment in the MS diet was also incremental in fashion but was significantly lower than the LS diet $24 \mathrm{~h}$ post exercise with glycogen replenished to $99.68 \%$ of resting values and $104.43 \%$, respectively. Again, the variations here can be readily explained by sample site error, the muscle biopsied, and fiber variation discussed previously. The rates of replenishment for both MS and LS were more rapid than previously reported (Pagan et al., 1987; Lacombe et al., 2004). However, they were similar to values reported by Vonderohe (2013), who reported the majority of horses replenished skeletal muscle glycogen at $24 \mathrm{~h}$.

Glycogen replenishment in the MS diet continued to rise, cumulating in the highest $48 \mathrm{~h}$ skeletal muscle glycogen concentration across diets at $10.59 \%$ over resting 
values. This result may indicate successful glycogen loading as described by Kline and Albert, (1981). Based on the results of the current study, glycogen loading was more effective in MS than HS.

Horses on the LS diet experienced a decrease in skeletal muscle glycogen concentration by $2.55 \%$ from $24 \mathrm{~h}$ to $48 \mathrm{~h}$ post exercise. This is interesting as the horses were not exercised between the $24 \mathrm{~h}$ and $48 \mathrm{~h}$ biopsies, although they were turned out in their normal housing. Vonderohe (2013) reported a larger depletion of $31.75 \%$ between $24 \mathrm{~h}$ and $48 \mathrm{~h}$ post biopsy for the LS diet. Additionally, Hyyppä et al. (1997) reported a depletion of $29.72 \%$ between $48 \mathrm{~h}$ and $72 \mathrm{~h}$ during a $3 \mathrm{~d}$ depletion study. This phenomena is best explained by the supercompensation effect seen at the $24 \mathrm{~h}$ sample. Due to a final rehydration and meal prior to the sample, an increase in the glycogen concentration occurred at $24 \mathrm{~h}$ (Vonderohe, 2013).

As previously discussed, there is a small variation in muscle fiber composition within the biceps femoris which may make it more metabolically active during recovery (Beecher et al., 1958; Snow and Guy, 1980). Also, given the location of the biceps femoris, locomotion may slightly restrict glycogen replenishment as exercise inhibits glycogen replenishment (Hodgson et al., 1983). The preferential replenishment of glycogen results in Type IIb fibers being replenished last, which may alter skeletal muscle glycogen concentration in these glycogen-rich fibers (Lindholm and Piehl, 1974; Snow et al., 1982). 


\section{Blood Lactate Concentration}

The production of lactic acid indicates anaerobic metabolism at high intensity exercise. The increase in blood lactate pre to post exercise indicates that the horses performed at an anaerobic level during the SET. There was no effect of treatment on lactate recovery, with very close concentrations experienced between diets $(\mathrm{P}=0.98)$. There was less variation between time points, which is indicative of horses maintaining, but not increasing, fitness throughout the project (Rainger et al., 1994). The similarities between the blood lactate concentrations between diets at various time points allowed for the formation of a prediction equation (Figure 8). Blood lactate clearance occurred in a linear fashion, which supported the findings of Marlin et al. (1991). The concentration of blood lactate decreases by approximately $54.7 \%$ from immediately post exercise to 10 min post exercise. By utilizing an identical SET, a subject should also experience this blood lactate clearance from post exercise to $10 \mathrm{~min}$. If a subject does not fit into the linear equation for the given SET, this may indicate that they are of a training status higher than the light working horses of the present study (Krzywanek et al., 1976; Snow and Mackenzie, 1977; Physick-Sheard, 1985). Alternatively, a subject completing the SET of the present study and having excessively high lactate concentrations may warrant veterinary observation. Failure for lactate clearance may indicate liver malfunction, as the liver stores lactate where it is converted into glucose though the Cori Cycle (Brooks et al., 2000). 


\section{Blood Glucose Concentration}

Energy is mobilized during high intensity exercise in the form of GLU, which is cleaved from glycogen in response to glucagon, adrenaline, and cortisol (Dent et al., 1990; Brooks, 1998). The significant spikes in GLU from rest to $0 \mathrm{~min}(\mathrm{P}<0.0001)$ would be expected due to the metabolic upregulation at the onset of exercise (Hyyppä, 2005). The positive correlations with skeletal muscle glycogen aligns with the increase in glycogen cleavage during exercise, resulting in GLU values higher 0 and 10 min post exercise than at rest and $24 \mathrm{~h}$ post exercise. Blood GLU concentration was not significant for MS or LS when comparing 0 min vs. $10 \min (P=0.16 ; P=0.86)$, respectively. However, blood GLU was significant for HS when comparing 0 min vs. 10 $\min (\mathrm{P}=0.009)$. This could be expected due to the higher glucose availability in a diet higher in starch (Lawrence, 1990; Hyyppä, 2005).

\section{Blood Urea Nitrogen Concentration}

Blood urea nitrogen measures the amount of blood nitrogen from the waste product urea. Urea is created in the liver when protein is broken down in the body. Blood urea nitrogen can be used to determine kidney function, as the kidney clears urea from the blood (Venes, 2009). Additionally, dehydration or a diet high in protein can cause elevated levels of BUN. There was a significant effect of time $(\mathrm{P}<0.0006)$ on BUN, with average levels rising from rest to 0 min post exercise across all diets. This rise is most likely due to protein breakdown during exercise as well as dehydration due to fluid losses (Tasker, 1965; Brooks et al., 2000; Waller et al., 2009b). 
There was a significant difference $(\mathrm{P}=0.03)$ between LS and HS. This may indicate that greater protein degradation occurred in LS (Graham-Thiers et al., 2003). Dietary fat was $6.9 \%$ and $13.6 \%$ for HS and LS, respectively. However, the low starch content in the LS diet may not have provided enough substrate for glycogen utilization during anaerobic exercise even with the sparing effects of fat (Lawrence, 1990; Pagan et al., 2015). This may be evidenced by the low levels of glycogen depletion in LS from rest to post exercise. The significantly higher concentration of BUN in LS could indicate that protein was utilized to fuel performance, as more AA were broken down for energy, thus increasing the waste product of ammonia and subsequently urea.

The significant difference between LS and HS BUN should not be solely due to dehydration. As evidenced by similar lactate level across treatments, work output and the need for thermoregulation would result in similar dehydration levels. However, fat supplementation has been shown to reduce heat production due to improvements in both aerobic and anaerobic performance (Scott et al., 1992; Kronfeld et al., 1994; Kronfeld, 2010). Kronfeld (2010) found that heat release was less in fat supplemented horses, resulting in less water needed for evaporative cooling. This would diminish the rate of whole body dehydration (Waller et al., 2009b).

\section{Conclusion}

Glycogen depletion and replenishment is significant in terms of fatigue and recovery to continue muscular work. The performance horse industry employs high intensity events over multiple days, which is of concern due to the slow rate horses replenish skeletal muscle glycogen stores. With a current industry trend of LS feeds, the 
importance of understanding the effects of LS on glycogen replenishment becomes prevalent. In the present study, feeding a HS diet with an average of $1,206.67 \mathrm{~g}$ starch/d did not yield an advantage in recovery time over a MS or LS diet. Horses on HS utilized greater glycogen amounts as evidenced through percentage total depletion, indicating reliance on $\mathrm{CHO}$ as the primary substrate for athletic performance.

The level of fat in LS is enough to elicit glycogen sparing during exercise. Additionally, horses on LS had greater BUN than HS, which anecdotally indicates subjects had greater reliance on protein as an energy source when on LS. This may indicate that horses fed on a LS diet for an extended period of time may break down muscle in order to fuel performance. Further exploration is needed to determine the consequences of feeding a diet low in starch to performance horses over a longer period of time.

The current study documented a SET that resulted in similar work output across various treatments, as observed by lactate accumulation. The prediction equation yielded by this study may aid in measuring fitness in equine subjects completing a SET identical to the present study.

This was the first study to compare a high, medium, and low starch diet with components that are commercially available and can be easily mixed by a person managing a horse's dietary needs. Adding SFC to a commercially available feed in quantities below the starch overload threshold allowed for greater substrate availability as evidenced by skeletal muscle glycogen depletion and GLU. However, further 
exploration is needed to determine the consequences of feeding low starch to performance horses. 


\section{LITERATURE CITED}

Alexander, F., and A. K. Chowdhury. 1958. Enzymes in the ileal juice of the horse. Nature. 181:190.

Armstrong, R. B., and R. O. Phelps. 1984. Muscle fiber type composition of the rat hindlimb. Am. J. Anat. 171:259-272.

Ashmore, C. R., G. Tompkins, and L. Doerr. 1972. Postnatal development of muscle fiber types in domestic animals. Jounal Anim. Sci. 34:37-41.

Baldwin, K. M., W. W. Winder, R. L. Terjung, and J. O. Holloszy. 1973. Glycolytic enzymes in different types of skeletal muscle: adaptation to exercise. Am. J. Physiol. 225:962-966.

Beecher, G. R., R. G. Cassens, W. G. Hoekstra, and E. J. Briskey. 1958. Red and white fiber content and associated post-mortem properties of seven porcine muscles. J. Food Sci. 30:969-976.

Bell, D. J. 1937. Glycogen: The molecular structure of horse muscle glycogen. Biochem. J. 31:1683-1691.

Bergström, J., L. Hermansen, and B. Saltin. 1967. Diet, muscle glycogen, and physical performance. Acta Physiol. Scand. 71:140-150.

Bergström, J., and E. Hultman. 1966. Muscle glycogen synthesis after exercise: an enhancing factor localized to the muscle cells in man. Nature:309-310.

Bergström, J., and E. Hultman. 1967. Synthesis of muscle glycogen in man after glucose and fructose infusion. Acta Med. Scand. 182:93-107.

Bergström, J. 1962. Muscle electrolytes in man determined by neutron activation analysis on needle biopsy specimens. Scand. J. Clin. Lab. Invest. 14:7-110.

Billman, G. E., J. X. Kang, and a Leaf. 1997. Prevention of ischemia-induced cardiac sudden death by n-3 polyunsaturated fatty acids in dogs. Lipids 32:1161-1168.

Brooks, G. A., T. D. Fahey, T. P. White, and K. M. Baldwin. 2000. Exercise physiology: human bioenergetics and its applications. 3rd ed. McGraw-Hill Higher Education, New York, NY.

Brooks, G. A. 1998. Mammalian fuel utilization during sustained exercise. Comp. Biochem. Physiol. Part B Biochem. Mol. Biol. 120:89-107. 
Burke, L. M., B. Kiens, and J. L. Ivy. 2004. Carbohydrates and fat for training and recovery. J. Sports Sci. 22:15-30.

Carrithers, J. A., D. L. Williamson, P. M. Gallagher, M. P. Godard, K. E. Schulze, and S. W. Trappe. 2000. Effects of postexercise carbohydrate-protein feedings on muscle glycogen restoration. J. Appl. Physiol. 88:1976-1982.

Casey, A., A. H. Short, E. Hultman, and P. L. Greenhaff. 1995. Glycogen resynthesis in human muscle fibre types following exercise-induced glycogen depletion. J. Physiol. 483:265-271.

Conlee, R. K., R. L. Hammer, W. W. Winder, M. L. Bracken, A. G. Nelson, and D. W. Barnett. 1990. Glycogen repletion and exercise endurance in rats adapted to a high fat diet. Metabolism. 39:289-294.

Davie, A. J., D. L. Evans, D. R. Hodgson, and R. J. Rose. 1994. The effects of an oral glucose polymer on muscle glycogen resynthesis in standardbred horses.

Davie, A. J., D. L. Evans, D. R. Hodgson, and R. J. Rose. 1995. Effects of intravenous dextrose infusion on muscle glycogen resynthesis after intense exercise. Equine Vet. J. 27:195-198.

Davie, A. J., D. L. Evans, D. R. Hodgson, and R. J. Rose. 1996. Effects of glycogen depletion on high intensity exercise performance and glycogen utilization rates. Pferdeheilkd. Sonderh. 12:482-484.

Davie, A. J., D. L. Evans, D. R. Hodgson, and R. J. Rose. 1999. Effects of Muscle Glycogen Depletion on Some Metabolic and Physiological Responses to Submaximal Treadmill Exercise. Can. J. Vet. Res. 63:241-247.

Dent, P., A. Lavoinne, S. Nakielny, F. B. Caudwell, P. Watt, and P. Cohen. 1990. The molecular mechanism bywhich insulin stimulates glycogen synthesis in mammalian skeletal muscle. Nature. 348:302-308.

Duren, S. E., S. G. Jackson, J. P. Baker, and D. K. Aaron. 1987. Effect of Dietary Fat on Blood Parameters in Exercised Thoroughbred Horses. In: J. Gillespie and N. E. Robinson, editors. Equine Exercise Physiology. ICEEP Publications, Davis, CA. p. 674-686.

Essén-Gustavsson, B., and A. Lindholm. 1985. Muscle fibre characteristics of active and inactive standardbred horses. Equine Vet. J. 17:434-438. 
Fell, R. D., J. A. McLane, W. W. Winder, and J. O. Holloszy. 1980. Preferential resynthesis of muscle glycogen in fasting rats after exhausting exercise. Am. J. Physiol. 238:R328-R332.

Geor, R. J. 2007. Perspectives on feeding athletic horses in the 21st century. In: Proceedings of the 5th Mid-Atlantic Nutrition Conference. p. 17-24.

Gollnick, P. D., R. B. Armstrong, B. Saltin, C. W. Saubert, W. L. Sembrowich, and R. E. Shepherd. 1973a. Effect of training on enzyme activity and fiber composition of human skeletal muscle. J.Appl.Physiol 34:107-111.

Gollnick, P. D., R. B. Armstrong, W. L. Sembrowich, R. E. Shepherd, and B. Saltin. 1973b. Glycogen depletion pattern in human skeletal muscle fibers after heavy exercise. J. Appl. Physiol. 34:615-618.

Gorostiaga, E. M., I. Navarro-Amézqueta, J. A. L. Calbet, L. Sánchez-Medina, R. Cusso, M. Guerrero, C. Granados, M. González-Izal, J. Ibáñez, and M. Izquierdo. 2014. Blood ammonia and lactate as markers of muscle metabolites during leg press exercise. J. Strength Cond. Res. 28:2775-2785.

Graham-Thiers, P. M., D. S. Kronfeld, K. A. Kline, D. J. Sklan, and P. A. Harris. 2003. Dietary protein and fat effects on protein status in Arabian horses during interval training and repeated sprints. J. Equine Vet. Sci. 23:554-559.

Greenhaff, P. L., J. B. Leiper, D. Ball, and R. J. Maughan. 1991. The influence of dietary manipulation on plasma ammonia accumulation during incremental exercise in man. Eur. J. Appl. Physiol. Occup. Physiol. 63:338-344.

Griewe, K. M., T. N. Meacham, and J. P. Fontenot. 1989. Effect of added dietary fat on exercising horses. In: Equine Nutrition and Physiology Society. Stillwater, OK. p. 101-106.

Guy, P. S., and D. H. Snow. 1977. The effect of training and detraining on lactate dehydrogenase isoenzymes in the horse. Biochem. Biophys. Res. Commun. 75:863-869.

Hambleton, P. L., L. M. Slade, D. W. Hamar, E. W. Kienholz, and L. D. Lewis. 1981. Dietary fat and exercise conditioning effect on metabolic parameters in the horse. J. Anim. Sci. 51:1330-1339. 
Harris, R. C., D. J. Marlin, and D. H. Snow. 1987. Metabolic response to maximal exercise of 800 and 2,000 $\mathrm{m}$ in the Thoroughbred horse. J. Appl. Physiol. 63:12 19.

Henneke, D. R., G. D. Potter, J. L. Kreider, and B. F. Yeates. 1983. Relationship between condition score, physical measurements and body fat percentage in mares. Equine Vet. J. 15:371-372.

Hodgson, D. R., C. M. McGowan, and K. H. McKeever. 2014. The Athletic Horse Principles and Practice of Equine Sports Medicine. 2nd ed. Elsevier Saunders, St. Louis, MO.

Hodgson, D. R., R. J. Rose, J. R. Allen, and J. Dimauro. 1985. Glycogen depletion patterns in horses competing in day 2 of a three day event. Cornell Vet. 75:366 74.

Hodgson, D. R., R. J. Rose, and J. R. Allen. 1983. Muscle glycogen depletion and repletion patterns in horses performing various distances of endurance exercise. Equine Exerc. Physiol.:229-236.

Hodgson, D. R. 2008. Assessment of performance: treadmill versus field techniques. In: 10th International Congress of World Equine Veterinary Association. IVIS, Moscow, Russia. p. 46-47.

Hoffman, R. M. 2009. Carbohydrate metabolism and metabolic disorders in horses. Rev. Bras. Zootec. 38:270-276.

Holloszy, J. O. 1967. Biochemical adaptations in muscle. J. Biol. Chem. 242:22782282.

Holmgren, A., and G. Ström. 1959. Blood lactate concentration in relation to absolute and relative work load in normal men, and in mitral stenosis, atrial septal defect and vasoregulatory asthenia. Acta Med. Scand. 163:185-193.

Horton, H. R., L. A. Moran, K. G. Scrimgeour, M. D. Perry, and J. D. Rawn. 2006. Metabolism and bioenergetics. In: Principles of Biochemistry. 4th ed. Pearson Prentice Hall, Upper Saddle River, NJ. p. 296-581.

Householder, D. D. 1978. Prececal, postileal and total tract digestion and growth performance in horses fed concentrate rations containing oats or sorghum grain processed by crimping or micronizing. Texas A\&M University.

Hurndall, J. S. 1896. Veterinary homeopathy in its application to the horse. Boericke and Tafel, Philadelphia, PA. 
Hyyppä, S., L. A. Räsänen, and A. R. Pösö. 1997. Resynthesis of glycogen in skeletal muscle from Standardbred trotters after repeated bouts of exercise. Am. J. Vet. Res. 58:162-166.

Hyyppä, S. 2005. Endocrinal responses in exercising horses. Livest. Prod. Sci. 92:113 121.

Ivy, J. L., M. C. Lee, J. T. Brozinick, and M. J. Reed. 1988. Muscle glycogen storage after different amounts of carbohydrate ingestion. J. Appl. Physiol. 65:2018 2023.

Jentjens, R., and A. E. Jeukendrup. 2003. Determinants of post exercise glycogen synthesis during short term recovery. Sport. Med. 33:117-144.

Jones, A. M., and J. H. Doust. 1996. A 1\% treadmill grade most accurately reflects the energetic cost of outdoor running. J. Sport. Sci. 14:321-7.

Jones, D. L., G. D. Potter, L. W. Greene, and T. W. Odom. 1992. Muscle glycogen in exercised miniature horses at various body conditions and fed a control or fat supplemented diet. J. Equine Vet. Sci. 12:287-291.

Jose-Cunilleras, E., K. W. Hinchcliff, V. A. Lacombe, R. A. Sams, C. W. Kohn, L. E. Taylor, and S. T. Devor. 2006. Ingestion of starch-rich meals after exercise increases glucose kinetics but fails to enhance muscle glycogen replenishment in horses. Vet. J. 171:468-477.

Jose-Cunilleras, E., and K. W. Hinchcliff. 2004. Carbohydrate metabolism in exercising horses. Equine Comp. Exerc. Physiol. 1:23-32.

Julen, T. R. 1994. Adaptation to a fat-supplemented diet by cutting horses. Texas A\&M University.

Julliand, V., A. de Fombelle, C. Drogoul, and E. Jacotot. 2001. Feeding and microbial disorders in horses: part 3-- Effects of three hay:grain ratios on microbial profile and activities. J. Equine Vet. Sci. 21:543-546.

Julliand, V., A. De Fombelle, and M. Varloud. 2006. Starch digestion in horses: the impact of feed processing. Livest. Sci. 100:44-52.

Kang, J. X. 2012. Reduction of heart rate by omega-3 fatty acids and the potential underlying mechanisms. Front. Physiol. 3:1-6. 
Kienzle, E., J. Pohlenz, and S. Radicke. 1997. Morphology of starch digestion in the horse. Zbl. Vetmed. A. 44:207-221.

Kienzle, E., S. Radicke, S. Wilke, E. Landes, and H. Meyer. 1992. Praeileal starch digestion in relation to source and preparation of starch. Eur. Konf. über die Ernährung des Pferdes, Hann.:103-106.

Kienzle, E. 1994. Small Intestinal Digestion of Starch in the Horse. Rev. Med. Vet. (Toulouse). 145.

Kitaoka, Y., Y. Endo, K. Mukai, H. Aida, A. Hiraga, and H. Hatta. 2014. Muscle glycogen breakdown and lactate metabolism during intensive exercise in Thoroughbred horses. J. Phys. Fit. Sport. Med. 3:451-456.

Kline, K. H., and W. W. Albert. 1981. Investigation of glycogen loading program for Standardbred horses. In: Seventh Equine Nutrition and Physiology Symposium. Warrenton, VA. p. 186-194.

Kreider, R. B., A. C. Fry, and M. L. O’Toole. 1997. Overtraining and overreaching in sport: terms, definitions, and prevalence. Human Kinetics Publishing, Champaign, IL.

Kronfeld, D. S., P. L. Ferrante, and D. Grandjean. 1994. Optimal nutrition for athletic performance, with emphasis on fat adaptation in dogs and horses. J. Nutr. 124:2745S-2753S.

Kronfeld, D. S. 2010. Dietary fat affects heat production and other variables of equine performance under hot and humid conditions. Equine Vet. J. 28:24-34.

Krzywanek, H., D. W. Milne, A. A. Gabel, and L. G. Smith. 1976. Acid-base values of Standardbred horses recovering from strenuous exercise. Am. J. Vet. Res. 37:291-294.

Lacombe, V. A., K. W. Hinchcliff, R. J. Geor, and M. A. Lauderdale. 1999. Exercise that induces substantial muscle glycogen depletion impairs subsequent anaerobic capacity. Equine Vet. J. Suppl. 30:293-297.

Lacombe, V. A., K. W. Hinchcliff, C. W. Kohn, S. T. Devor, and L. E. Taylor. 2004. Effects of feeding meals with various soluble-carbohydrate content on muscle glycogen synthesis after exercise in horses. Am. J. Vet. Res. 65:916-923. 
Larsson, Y., B. Persson, G. Sterky, and C. Thorén. 1964. Functional adaptation to rigorous training and exercise in diabetic and nondiabetic adolescents. J. Appl. Physiol. 19:629-635.

Lawrence, L. M. 1990. Nutrition and fuel utilization in the athletic horse. Vet. Clin. North Am. Equine Pract. 6:393-418.

Lemon, P. W. 1987. Protein and exercise: update 1987. Med. Sci. Sports Exerc. 19:S179-S190.

Lindholm, A., H. Bjerneld, and B. Saltin. 1974. Glycogen depletion pattern in muscle fibres of trotting horses. Acta Physiol. Scand. 90:475-484.

Lindholm, A., and K. Piehl. 1974. Fibre composition, enzyme activity and concentrations of metabolites and electrolytes in muscles of Standardbred horses. Acta Vet. Scand. 15:287-309.

Lindholm, A., and B. Saltin. 1974. The physiological and biochemical response of Standardbred horses to exercise of varying speed and duration. Acta Vet. Scand. 15:310-324.

Marlin, D. J., R. C. Harris, and D. H. Snow. 1991. Rates of blood lactate disappearance following exercise of different intensities. 3:188-195.

McCutcheon, L. J., R. J. Geor, and K. W. Hinchcliff. 1999. Effects of prior exercise on muscle metabolism during sprint exercise in horses. J. Appl. Physiol. 87:1914 1922.

McGowan, C. M., and D. J. Whitworth. 2008. Overtraining syndrome in horses. Comp. Exerc. Physiol. 5:57-65.

Meyers, M. C., G. D. Potter, J. W. Evans, L. W. Greene, and S. F. Crouse. 1989. Physiologic and metabolic response of exercising horses to added dietary fat. In: Journal of Equine Veterinary Science. Vol. 9. p. 218-223.

Miller, P. A., and L. M. Lawrence. 1986. Changes in equine metabolic characteristics due to exercise fatigue. Am. J. Vet. Res. 47:2184-2186.

Nielsen, J., H.-C. Holmberg, H. D. Schrøder, B. Saltin, and N. Ortenblad. 2011. Human skeletal muscle glycogen utilization in exhaustive exercise: role of subcellular localization and fibre type. J. Physiol. 589:2871-2885. 
Nimmo, M. A., and D. H. Snow. 1983. Changes in muscle glycogen, lactate, and pyruvate concentrations in the Thoroughbred horse following maximal exercise. Equine Exerc. Physiol.:237-244.

NRC. 2007. Nutrient Requirements of Horses. 6th Revise. The National Academies Press.

Oldham, S. L., G. D. Potter, J. W. Evans, S. B. Smith, T. S. Taylor, and W. S. Barnes. 1990. Storage and mobilization of muscle glycogen in exercising horses fed a fat supplemented diet. J. Equine Vet. Sci. 10:353-359.

Pagan, J. D., B. Essén-Gustavsson, A. Lindholm, and R. Thornton. 1987. The effect of dietary energy source on exercise performance in Standardbred horses. Equine Exerc. Physiol.:686-700.

Pagan, J. D., V. S. Mesquita, S. J. Valberg, B. Waldridge, and C. Whitehouse. 2015. Effect of non-structural carbohydrate intake on glycogen repletion following intense exercise. J. Equine Vet. Sci. 35:408-409.

Pagan, J. D. 1998. Energy and the performance horse. 141-148.

Peoples, G. E., P. L. McLennan, P. R. C. Howe, and H. Groeller. 2008. Fish oil reduces heart rate and oxygen consumption during exercise. J. Cardiovasc. Pharmacol. 52:540-547.

Physick-Sheard, P. W. 1985. Cardiovascular response to exercise and training in the horse. Vet. Clin. North Am. Equine Pract. 1:383-417.

Poole, D. C., and H. H. Erickson. 2004. Heart and vessels: function during exercise and response to training. J. Equine Vet. Sci. 24:288-294.

Potter, G. D., F. F. Arnold, D. D. Householder, and D. H. Hansen. 1992. Digestion of starch in the small or large intestine of the equine. Pferdeheilkd. Sonderh.

Rainger, J. E., D. L. Evans, D. R. Hodgson, and R. J. Rose. 1994. Blood lactate disappearance after maximal exercise in trained and detrained horses. Res. Vet. Sci. 57:325-331.

Reynolds, A. J., D. P. Carey, G. A. Reinhart, R. A. Swenson, and F. A. Kallfelz. 1997. Effect of postexercise carbohydrate supplementation on muscle glycogen repletion in trained sled dogs. Am. J. Vet. Res. 58:1252-1256. 
Robinson, S., and P. M. Harmon. 1941. The lactic acid mechanism and certain properties of the blood in relation to training. Am. J. Physiol. 132:757-769.

Rottmann, J. 1994. Untersuchungen zur Verdaulichkeit (insgesamt und praeileal) von \ Maissilage und Maiskolbensilage beim Pferd. Tierärztliche Hochschule Hannover.

Saitoh, S., Y. Tasaki, K. Tagami, and M. Suzuki. 1994. Muscle glycogen repletion and pre-exercise glycogen content: effect of carbohydrate loading in rats previously fed a high fat diet. Eur. J. Appl. Physiol. Occup. Physiol. 483-488.

Saltin, B., J. Henriksson, E. Nygaard, P. Andersen, and E. Jansson. 1977. Fiber types and metabolic potentials of skeletal muscles in sedentary man and endurance runners. Ann. N. Y. Acad. Sci. 301:3-29.

Schuback, K., and B. Essén-Gustavsson. 1998. Muscle anaerobic response to a maximal treadmill exercise test in Standardbred trotters. Equine Vet. J. 30:504-510.

Scott, B. D., G. D. Potter, L. W. Greene, E. Hargis, and J. G. Anderson. 1992. Efficacy of a fat-supplemented diet on muscle glycogen concentrations in exercising Thoroughbred horses maintained in varying body conditions. In: Equine Nutrition and Physiology Society. Vol. 12. p. 109-113.

Seeherman, H. J., and E. A. Morris. 1990. Methodology and repeatability of a standardized treadmill exercise test for clinicical evaluation of fitness in horses. Equine Vet. J. 22:20-25.

Shirazi-Beechey, S. P. 1995. Molecular Biology of Intestinal Glucose Transport. Nutr. Res. Rev. 8:27-41.

Sigler, D. H. 1981. Relationship of muscle fiber type and metabolism in Quarter Horses to varying exercise regimens. Texas A\&M Unviersity.

Snow, D. H., and P. S. Guy. 1976. Percutaneous needle muscle biopsy in the horse. Equine Vet. J. 8:150-155.

Snow, D. H., and P. S. Guy. 1980. Muscle fibre type composition of a number of limb muscles in different types of horse. Res. Vet. Sci. 28:137-144.

Snow, D. H., and R. C. Harris. 1991. Effects of daily exercise on muscle glycogen in the Thoroughbred racehorse. Equine Exerc. Physiol. 3:299-304. 
Snow, D. H., M. G. Kerr, M. A. Nimmo, and E. M. Abbott. 1982. Alterations in blood, sweat, urine and muscle composition during prolonged exercise in the horse. Vet. Rec. 110:377-84.

Snow, D. H., and G. Mackenzie. 1977. Some metabolic effects of maximal exercise in the horse and adaptations with training. Equine Vet. J. 9:134-140.

Spencer, M. K., Z. Yan, and A. Katz. 1992. Effect of low glycogen on carbohydrate and energy metabolism in human muscle during exercise. Am. J. Physiol. 262:C975 C979.

Tarnopolsky, M. A., E. Pearce, K. Smith, and B. Lach. 2011. Suction-modified Bergström muscle biopsy technique: experience with 13,500 procedures. Muscle Nerve 43:716-725.

Tasker, J. B. 1965. Fluid and electrolyte studies in the horse: blood values in 100 normal horses. Cornell Vet. 56:67-67.

Tesch, P. 1980. Muscle fatigue in man with special reference to lactate accumulation during short term intense exercise. Acta Physiol. Scand. Suppl. 480:1-40.

Thorstensson, A., B. Sjödin, and J. Karlsson. 1975. Enzyme activities and muscle strength after "sprint training" in man. Acta Physiol. Scand. 94:313-318.

Topliff, D. R. 1984. Diet manipulation, muscle glycogen metabolism, and work performance in the equine. Texas A\&M.

Valberg, S. 1986. Glycogen depletion patterns in the muscle of standardbred trotters after exercise of varying intensities and durations. Equine Vet. J. 18:479-484.

Van Loon, L. J. C., W. H. M. Saris, M. Kruijshoop, and A. J. M. Wagenmakers. 2000. Maximizing postexercise muscle glycogen synthesis: Carbohydrate supplementation and the application of amino acid or protein hydrolysate mixtures. Am. J. Clin. Nutr. 72:106-111.

Varloud, M., A. De Fombelle, A. G. Goachet, C. Drogoul, and V. Julliand. 2004. Partial and total apparent digestibility of dietary carbohydrates in horses as affected by the diet. Anim. Sci. 79:61-72.

Venes, D. 2009. Taber's Cyclopedic Medical Dictionary. 21st ed. F. A. Davis Company, Philadelphia, PA. 
Vonderohe, C. E. 2013. The effect of dietary starch concentration on glycogen replenishment in performance horses. Texas A\&M University.

Waller, A. P., R. J. Geor, L. L. Spriet, G. J. F. Heigenhauser, and M. I. Lindinger. 2009a. Oral acetate supplementation after prolonged moderate intensity exercise enhances early muscle glycogen resynthesis in horses. Exp. Physiol. 94:888-898.

Waller, A. P., G. J. F. Heigenhauser, R. J. Geor, L. L. Spriet, and M. I. Lindinger. 2009 b. Fluid and electrolyte supplementation after prolonged moderate-intensity exercise enhances muscle glycogen resynthesis in Standardbred horses. J. Appl. Physiol. 106:91-100.

Waller, A. P., and M. I. Lindinger. 2010. Nutritional aspects of post exercise skeletal muscle glycogen synthesis in horses: a comparative review. Equine Vet. J. 42:274-281.

Wasserman, K., and B. Whipp. 1975. Exercise physiology in health and disease. Am. Rev. Respir. Dis. 112:219-249.

Zawadzki, K. M., B. B. Yaspelkis, and J. L. Ivy. 1992. Carbohydrate-protein complex increases the rate of muscle glycogen storage after exercise. J. Appl. Physiol. 72:1854-1859. 


\section{APPENDIX A}

\section{Skeletal Muscle Glycogen Concentration Initial Incubation Statistical Analysis}

The average skeletal muscle glycogen concentrations ( $\mu \mathrm{g} / \mathrm{mg}$ wet weight) for the initial incubation period taken at rest, time $0,24 \mathrm{~h}$, and $48 \mathrm{~h}$ post SET are presented in Table 9 and Figure 3. There was a significant effect on period $(P=0.01)$ and treatment $(P=0.04)$. The differences of least squared means showed a significant $(P=0.01)$ difference between LS and MS in terms of average glycogen concentration. When period effect was taken into consideration, DLSM was utilized to further analyze treatment $\mathrm{x}$ time interactions. There was a significant difference between $\mathrm{HS}$ and MS at rest $(\mathrm{P}=$ $0.008)$ and $\mathrm{LS}$ and MS at $24 \mathrm{~h}$ post SET $(\mathrm{P}=0.01)$. 


\section{APPENDIX B}

Table 15. Mean Skeletal Muscle Glycogen Concentration ( $\mu \mathrm{g} / \mathrm{mg}$ wet weight) Initial Incubation $\left( \pm \mathrm{SE}^{*}\right)$

Table 15. Mean Skeletal Muscle Glycogen Concentration ( $\mu \mathrm{g} / \mathrm{mg}$ wet weight) Initial Incubation $\left( \pm \mathrm{SE}^{*}\right)$

\begin{tabular}{l|c|c|c|c|c|c|}
\hline \multicolumn{3}{|c}{ High Starch $^{12}$} & \multicolumn{3}{c}{ Medium Starch $^{2}$} & \multicolumn{2}{c}{ Low Starch $^{\mathbf{1}}$} \\
\hline $\begin{array}{l}\text { Sample } \\
\text { Time }\end{array}$ & Concentration & SE* & Concentration & SE* & Concentration & SE* $^{*}$ \\
\hline Rest & $11.37^{\mathrm{a} \dagger}$ & 1.14 & $8.74^{\mathrm{a} \dagger}$ & 1.12 & $10.50^{\mathrm{a}}$ & 1.00 \\
\hline $\begin{array}{l}\text { Post } \\
\text { Exercise }\end{array}$ & $8.38^{\mathrm{b}}$ & 0.66 & $8.56^{\mathrm{a}}$ & 0.36 & $9.83^{\mathrm{a}}$ & 0.80 \\
\hline $24 \mathrm{~h}$ & $8.62^{\mathrm{b}}$ & 0.97 & $8.33^{\mathrm{a} \dagger}$ & 0.75 & $10.66^{\mathrm{a} \dagger}$ & 0.90 \\
\hline $48 \mathrm{~h}$ & $9.90^{\mathrm{ab}}$ & 0.67 & $9.73^{\mathrm{a}}$ & 0.59 & $9.19^{\mathrm{a}}$ & 0.61 \\
\hline
\end{tabular}

*SE $=$ Standard error within diet

${ }^{1,2}$ Diets not sharing the same superscript differ $(\mathrm{P}<0.05)(\mathrm{SE} \pm 0.67)$

${ }^{\mathrm{a}, \mathrm{b}}$ Values in the same column not sharing the same superscript differ $(\mathrm{P}<0.05)$

$\dagger$ Values in the same row differ $(\mathrm{P}<0.05)$ 


\section{APPENDIX C}

Figure 7. Mean Skeletal Muscle Glycogen Concentration Initial Incubation $\left( \pm \mathrm{SE}^{*}\right)$

Figure 7. Mean Skeletal Muscle Glycogen Concentration

Initial Incubation $\left( \pm \mathrm{SE}^{*}\right)$

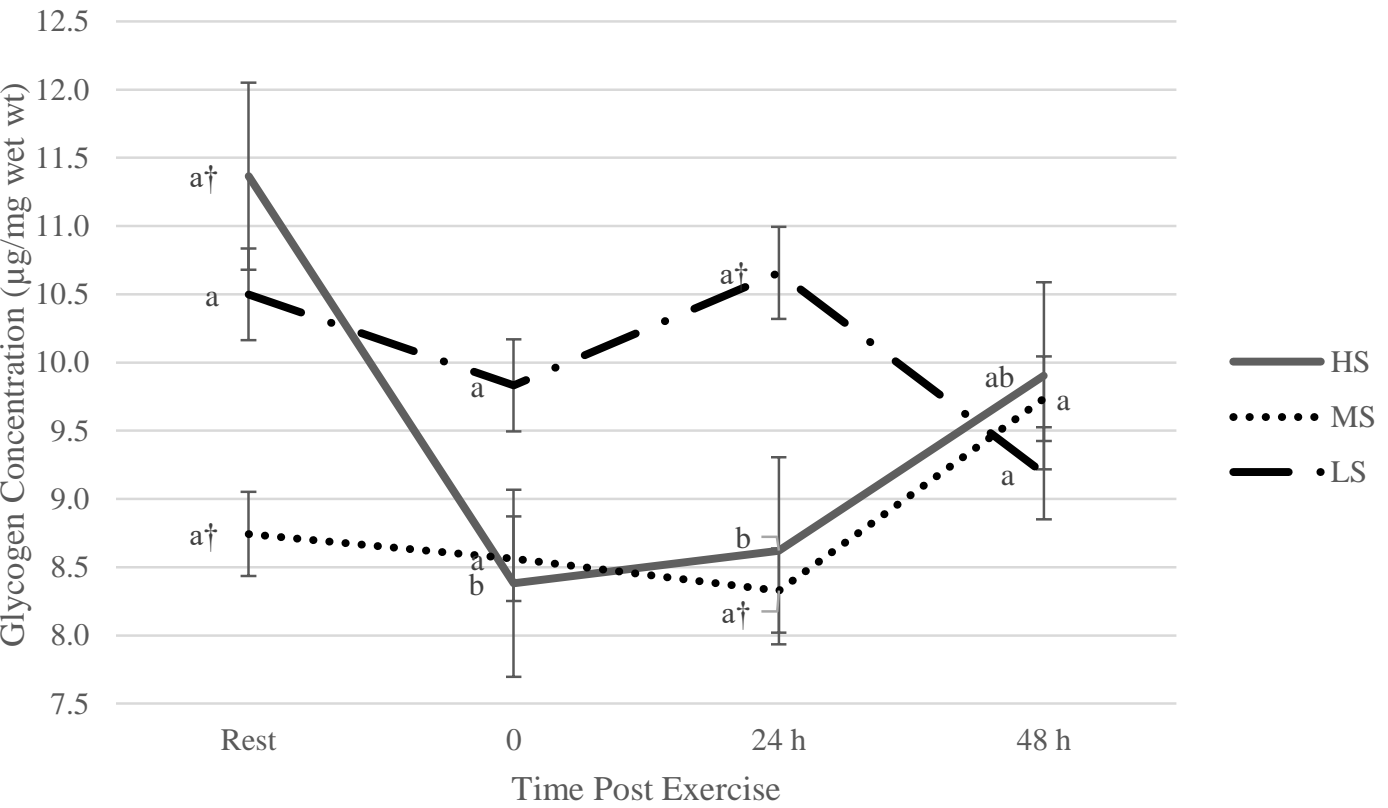

$* \mathrm{SE}=$ Standard error

a,bValues in the same diet not sharing the same superscript differ $(\mathrm{P}<0.05)$

$\uparrow$ Values at the same time point differ $(\mathrm{P}<0.05)$ 


\section{APPENDIX D}

\section{Mean Skeletal Muscle Glycogen Percentage Depletion/Repletion Initial Incubation}

Table 16. Mean Skeletal Muscle Glycogen Percentage Depletion/Repletion Initial Incubation

\begin{tabular}{l|c|c|c|c|c|c}
\hline \multicolumn{2}{c}{ High Starch } & \multicolumn{5}{c}{ Medium Starch } \\
Sample \\
$\begin{array}{l}\text { Time } \\
\text { Time of }\end{array}$ & $\begin{array}{c}\% \\
\text { Rest } \\
\text { Value }\end{array}$ & $\begin{array}{c}\text { Change } \\
\text { Value }\end{array}$ & $\begin{array}{c}\% \\
\text { Change }\end{array}$ & $\begin{array}{c}\text { \% of Rest } \\
\text { Value }\end{array}$ & \% Change \\
\hline Rest & $100^{\mathrm{a} \dagger}$ & 0 & $100^{\mathrm{a} \dagger}$ & 0 & $100^{\mathrm{a}}$ & 0 \\
\hline $\begin{array}{l}\text { Post } \\
\text { Exercise }\end{array}$ & $73.75^{\mathrm{b}}$ & 26.25 & $97.92^{\mathrm{a}}$ & 2.08 & $93.65^{\mathrm{a}}$ & 6.35 \\
\hline $24 \mathrm{~h}$ & $75.85^{\mathrm{b}}$ & 24.15 & $95.24^{\mathrm{a} \dagger}$ & 4.76 & $101.5^{\mathrm{a} \dagger}$ & -1.15 \\
\hline $48 \mathrm{~h}$ & $87.14^{\mathrm{ab}}$ & 12.86 & $111.33^{\mathrm{a}}$ & -11.33 & $87.52^{\mathrm{a}}$ & 12.48 \\
\hline
\end{tabular}

${ }^{1,2}$ Diets not sharing the same superscript differ $(\mathrm{P}<0.05)(\mathrm{SE} \pm 0.67)$

${ }^{a, b}$ Values in the same column not sharing the same superscript differ $(\mathrm{P}<0.05)$

$\dagger$ Values in the same row differ $(\mathrm{P}<0.05)$ 


\section{APPENDIX E}

Figure 8. Glycogen Percentage Depletion/Repletion Initial Incubation $\left( \pm \mathrm{SE}^{*}\right)$

Figure 8. Glycogen Percentage Depletion/Repletion Initial Incubation $\left( \pm \mathrm{SE}^{*}\right)$

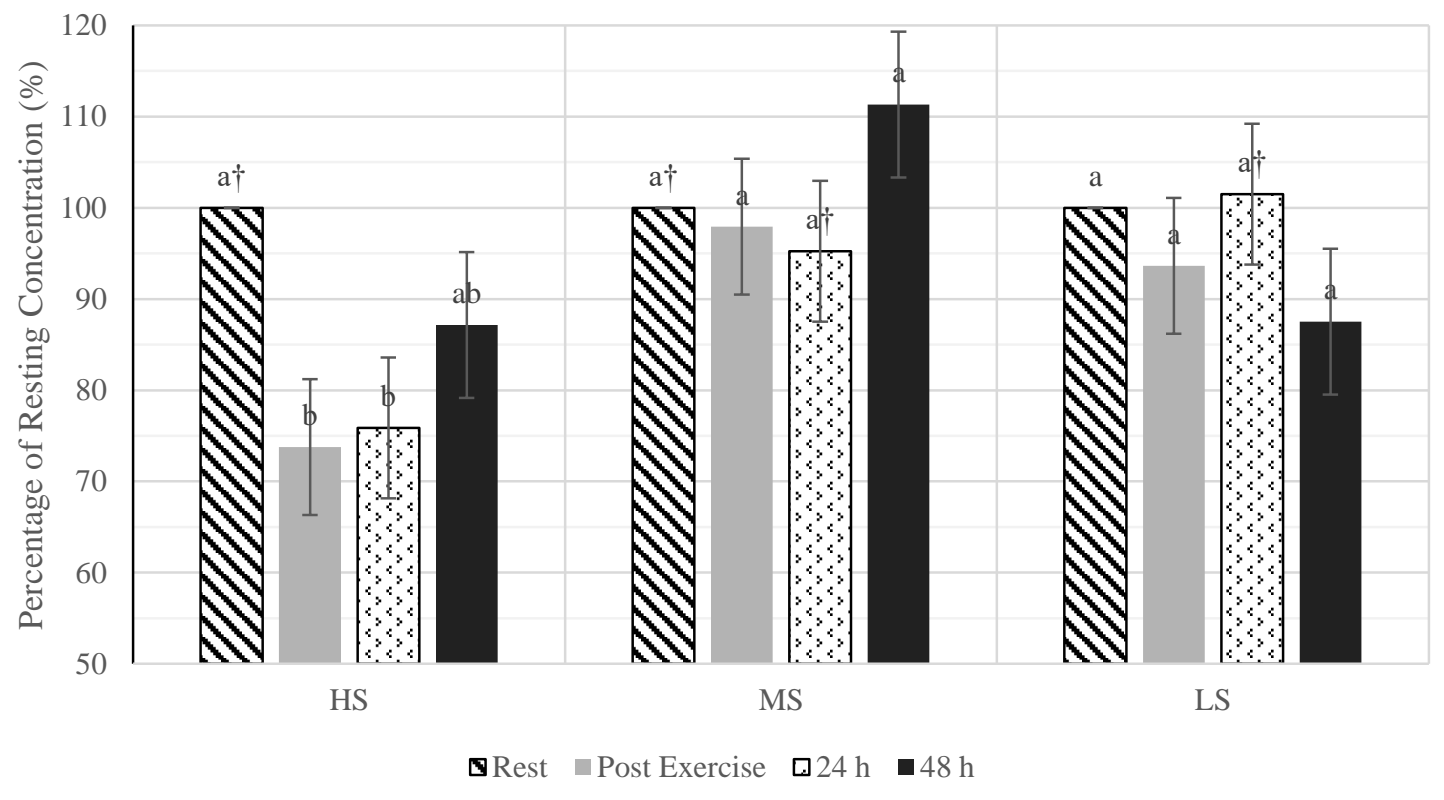

$* \mathrm{SE}=$ Standard error

a,b Values in the same diet not sharing the same superscript differ $(\mathrm{P}<0.05)$

$\uparrow$ Values at the same time point differ $(\mathrm{P}<0.05)$ 


\section{APPENDIX F}

\section{Analysis of Glycogen Percentage Depletion/Repletion Initial Incubation}

High starch had the greatest depletion of glycogen post exercise with $26.25 \%$.

High starch repleted $2.1 \%$ from post exercise to $24 \mathrm{~h}$ post (difference between percentage changes of post and 24 h). High starch repleted $11.29 \%$ between 24 and 48

h. Medium starch had the lowest depletion of glycogen post exercise with $2.08 \%$. Medium starch depleted from post exercise to 24 h by $2.68 \%$. Medium starch experienced the greatest replenishment of $16.09 \%$ and had an $11.33 \%$ greater glycogen concentration $48 \mathrm{~h}$ post exercise than at rest. Low starch had a $6.35 \%$ glycogen depletion from rest to post exercise. Low starch repleted glycogen from post exercise to 24 h by $7.5 \%$, resulting in an increase in glycogen concentration $1.15 \%$ over resting glycogen levels at $24 \mathrm{~h}$. Between 24 and 48 h, LS depleted further by $13.63 \%$, resulting in a glycogen concentration $12.48 \%$ lower than the resting glycogen concentration.

Horses on the HS diet utilized the greatest amount of glycogen. The MS diet resulted in the smallest amount of glycogen depletion. Low starch experienced a period of rapid depletion between 24 and 48 h post. The MS diet resulted in the greatest concentration of glycogen $48 \mathrm{~h}$ post exercise as compared to resting concentration. HS and LS experienced similar glycogen replenishment of $87.14 \%$ and $87.52 \%$ of the resting concentration, respectively. The percentage of skeletal muscle glycogen at $48 \mathrm{~h}$ compared to resting concentration was MS > LS > HS. 